\title{
Preventing Sybil Attacks by Privilege Attenuation: A Design Principle for Social Network Systems
}

\author{
Philip W. L. Fong \\ Department of Computer Science \\ University of Calgary \\ Calgary, Alberta, Canada \\ Email: pwlfong@ucalgary.ca
}

\begin{abstract}
In Facebook-style Social Network Systems (FSNSs), which are a generalization of the access control model of Facebook, an access control policy specifies a graph-theoretic relationship between the resource owner and resource accessor that must hold in the social graph in order for access to be granted. Pseudonymous identities may collude to alter the topology of the social graph and gain access that would otherwise be forbidden. We formalize Denning's Principle of Privilege Attenuation (POPA) as a run-time property, and demonstrate that it is a necessary and sufficient condition for preventing the above form of Sybil attacks. A static policy analysis is then devised for verifying that an FSNS is POPA compliant (and thus Sybil free). The static analysis is proven to be both sound and complete. We also extend our analysis to cover a peculiar feature of FSNS, namely, what Fong et al. dubbed as Stage-I Authorization. We discuss the anomalies resulted from this extension, and point out the need to redesign Stage-I Authorization to support a rational POPA-compliance analysis.
\end{abstract}

Keywords-access control; policy analysis; social network systems; Sybil attacks; Principle of Privilege Attenuation; soundness and completeness of static analysis.

\section{INTRODUCTION}

One way of gauging the effectiveness of an access control scheme is by carefully articulating the security property that is enforced by the scheme. For example, by showing that a protection mechanism has the noninterference property [1], [2], one knows that the high-level information flow policy is indeed enforced by the mechanism. When the target security property is ambiguous, the access control mechanism adds guards and mediations with no clear security goal.

The rise of social computing introduces the world to a new paradigm of access control, in which the inter-personal relationships between users are explicitly articulated in a social graph that forms the basis of authorization decisions. Authors have begun to refer to this emerging paradigm as RelationshipBased Access Control (ReBAC) [3], [4], [5], [6]. For instance, Fong et al. [7], [8] generalized the access control mechanism of the popular Social Network System (SNS), Facebook, into a family of access control systems known as Facebook-style Social Network Systems (FSNSs). A distinctive feature of FSNSs is that every access control policy specifies a graphtheoretic relationship between the resource owner and the resource accessor (e.g., the owner and accessor share $k$ or more common friends). Access is granted when the stated relationship is realized in the social graph. A question one must ask is the following: Exactly what is the security goal of such a protection paradigm? That is, what security property is enforced by this protection paradigm? This work is an attempt to answer the questions above.

We demonstrate that some FSNSs, when improperly configured, are amenable to Sybil attacks [9]. In a classical Sybil attack, a malicious user of a peer-to-peer system creates multiple pseudonymous identities, and uses their combined influence to bypass the reputation system. In an FSNS, because authorization decision is a function of the current topology of the social graph, it is possible that a group of pseudonymous identities may collude to manipulate the topology of the social graph, with the effect of gaining privileges that would otherwise be forbidden. (In this work, we concern ourselves with "formal" Sybil attacks, in which malicious users add or remove edges in the social graph without employing social engineering. Sybil attacks involving social engineering, such as Profile Cloning attacks [10], [11], [12], are beyond the scope of this work.) The prevention of Sybil attacks is taken to be a security goal of FSNSs in this work.

The articulation of the above security goal begs the question of whether Sybil attacks can be prevented by a properly designed FSNS. In early work on access control systems, Denning formulated a design principle, the Principle of Privilege Attenuation (POPA), to prevent privilege escalation caused by the collusion of unprivileged users. In short, POPA demands that a user may confer a privilege only when she already has that privilege. It turns out that POPA can be adopted in the design of FSNSs to effectively prevent Sybil attacks. Specifically, this work is the first one to propose a policy analysis that will allow an FSNS designer to statically certify that the FSNS complies to POPA, and thus provably prevent Sybil attacks at run time. Specifically, the following are the contributions of this work:

1) We formalize POPA as a property of the set of execution traces generated by an FSNS, in a manner akin to noninterference [1], [2]. We show that this formulation of POPA is a necessary and sufficient condition for preventing Sybil attacks in FSNSs.

2) We define a static analysis that allows one to examine only the current configuration (i.e., privacy settings) of the FSNS, and determine if the FSNS complies to POPA. We prove that the static analysis is sound: if 
the current configuration of the FSNS passes the static analysis, then Sybil attacks will not occur at run time. This result can be further exploited so that an FSNS designer can statically analyze the vocabulary of policies supported by the FSNS, and certify the FSNS to be POPA compliant under every possible configuration.

3) We show that the above static analysis is complete: if the configuration of the FSNS complies to POPA, then the static analysis will recognize it as such. This means the analysis is precise.

4) We extend the static analysis to cover a peculiar feature of FSNS. In an FSNS, a necessary condition for access is that the accessor must "reach" the "search listing" of the profile owner (see Section II-A for more details). We illustrate the complexities and anomalies that will arise from widening the scope of the policy analysis to cover this feature. This motivates a need to redesign the access control mechanism of FSNSs to facilitate POPAcompliance analysis.

\section{OVERVIEW}

The kind of Sybil attacks studied in this work is distinct from its counterparts in peer-to-peer systems [13], [14], reputation systems [15] and recommendation systems [16]. Sybil attack prevention in FSNSs is a novel research challenge that has not been studied in the past. This section gives an overview of this research problem and our solution approach.

\section{A. Facebook-style Social Network Systems}

We review here an analysis of Facebook's access control mechanism that was reported by Fong et al. [7]. We then outline a family of SNSs that they identified to be Facebookstyle Social Network Systems.

1) Profile and Profile Items: Facebook allows each user to construct a representation of herself in the form of a profile. A profile displays such profile items as personal information (e.g., favorite books), multimedia contents (e.g., pictures), activity $\operatorname{logs}$ (e.g., status), or other user-authored contents (e.g., blog-like postings). Facebook users may grant one another access to the profile items they own.

2) Search Listings and their Reachability: Access to profile items is authorized in two stages. In Stage I, the accessing user must reach the search listing of the profile owner. Then in Stage II, the accessing user requests access to the profile, and the profile items are selectively displayed. The search listing of a user could be seen as a "capability" [17], [18] of the user in the system, through which access is mediated. There are two means by which a search listing may be reached in Stage I - global name search and social graph traversal.

3) Global Name Search: A successful global name search produces for the accessing user the search listing of the target user. A profile owner may specify a search policy to permit only a subset of users to reach her search listing through a global name search.
4) Social Graph Traversal: A second means to reach a search listing is by traversing the social graph. Facebook allows users to articulate their relationships with one another through the construction of friend lists. Every user may list a set of other users as her friends. As friendship is an irreflexive, symmetric binary relation, it induces a simple graph known as the social graph, in which users are vertices and relationships are edges. A user may traverse this graph by examining the friend lists of other users. A user $u$ may restrict traversal by specifying a traversal policy, which specifies the set of users $v$ who are allowed to examine $u$ 's friend list after $v$ has reached the search listing of $u$.

5) Profile Access: Once the search listing of a profile owner is reached, the accessing user may elect to access the profile, thereby initiating Stage II of authorization. Not every accessing user sees the same profile items when a profile is displayed. The owner may assign an access policy to each profile item, dictating who can see that profile item when the profile is accessed. This is the means through which a user may project different representations of herself to different groups of users.

6) Friendship Articulation: Articulating friendship involves a consent protocol, whereby a user sends a friendship invitation to another user, who may then accept or ignore the invitation. Once a mutual consent is reached, that friendship is recorded by Facebook as an edge in the social graph. Such an operation is called befriending. Befriending invitation is guarded in Facebook. To initiate a befriending invitation, the inviting user must first reach the search listing of the invited user (i.e., Stage I). Then, the acquaintance policy of the invited user will determine if the inviting user can extend the befriending invitation. On the other hand, defriending, that is, the dissolution of friendship, is not guarded. It can be carried out unilaterally by either end of the relationship.

7) Policies: We have seen in the above discussion that various aspects of user activities are controlled by userspecified policies (e.g., search policy, access policy, etc). This is typical of a discretionary access control system [19], [20], in which a user may grant access privileges to other users. Facebook offers a fixed vocabulary of predefined policies for users to choose from when they are to identify sets of privileged users. These predefined policies identify user sets not in terms of user identities, but rather in terms of how the accessor and the owner are related to one another in the social graph. For example, one may specify that a certain profile item is accessible only by "friends" of the profile owner, or that befriending invitation is only available to "friends of friends".

8) Facebook-style Social Network Systems: Fong et al. [7] proposed a generalization of the above access control mechanism, whereby the policy vocabulary may contain policies that specify complex graph-theoretic relationships between the owner and the accessor. For example, the policy vocabulary of such an SNS may contain a policy that grants access when the owner and the accessor share $k$ common friends (i.e., "friends of friends" is a special case with $k=1$ ). As another example, consider a policy that grants access when 


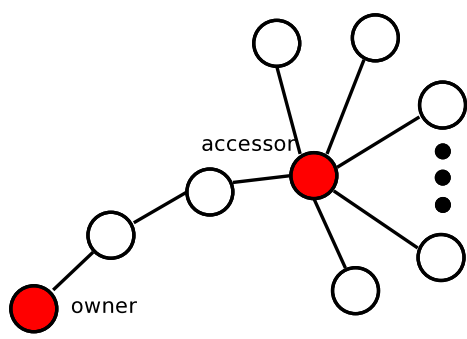

Fig. 1. "Somewhat related" and "popular."

the owner and the accessor belong to the same $k$-clique (i.e., "friends" is a special case when $k=2$ ). A Facebook-style Social Network System (FSNS) is an SNS that shares with Facebook the same access control mechanism as described above, but with its own vocabulary of policies. The family of FSNSs effectively specifies a design space for Facebook-like SNSs, so that the design space is parameterized by the choice of policy vocabulary, where Facebook is but one point of the design space. The goal of this work is to identify a design criterion for selecting a "good" policy vocabulary.

\section{B. Sybil Attacks in FSNSS}

The normal assumption of a SNS is that every user is represented by exactly one user account (i.e., one identity), and thus each vertex of the social graph is controlled by a distinct user. Typically this assumption is enforced by imposing terms of use, by CAPTCHA, and by cross-referencing personal data collected from users. There is, however, no "hard" mechanism for strictly enforcing such a policy. It is entirely possible for a user to assume multiple identities [10], [11], [12]. The result is that the same human user could offer befriending or defriending consent on behalf of multiple social graph vertices.

In FSNSs, accessibility is a function of the topology of the social graph. By taking control of multiple user accounts, and strategically initiating befriending and defriending actions, a user can manipulate the topology of the social graph neighbourhood that is dominated by the vertices she controls. A Sybil attack [9] is resulted when a malicious user, through setting up multiple accounts, coordinates the addition and deletion of edges in the social graph, in such a manner that allows her to gain privileges that otherwise she would not possess.

Example 1. Suppose an owner allows access to her datingready photo only if the accessor is "somewhat related", and is a "popular" person. What is an access policy that appropriately represents this privacy need? To ensure the accessor is "somewhat related", let us demand the accessor to be of distance no further than three from the owner. To ensure the accessor is "popular", let us further demand the accessor to have a vertex degree of one hundred or more (Figure 1).

Now suppose there is an attacker who is "somewhat related" (distance no more than three), but not "popular" enough (degree less than a hundred). How can the attacker launch a Sybil attack to gain access to the owner's photo? All she needs to do is to create enough fake accounts, and befriend them accordingly. As she controls both her original account and these fake accounts, she could offer consent to any befriending requests.

Notice that the attack above can be launched in a slightly different manner. Suppose the attacker does not create fake accounts, but instead colludes with an appropriate number of strangers by befriending them. She will then be able to increase her vertex degree, and thus gain access as a result. This observation leads to a core insight we would like to highlight:

In FSNSs, there is no material difference between

Sybil attacks and the collusion of unprivileged users

to gain access.

Creation of pseudonymous identities is not the crux of the problem. The crux of the problem is whether an SNS can be configured to prevent unprivileged users from manipulating the access control system into conferring to them privileges that they do not rightly possess.

\section{Principle of Privilege Attenuation}

In early work on access control systems, Denning formulated a design principle to avoid exactly this kind of problems. Specifically, to prevent unprivileged users from colluding with one another to gain access, Denning advocates the Principle of Privilege Attenuation (POPA) [21, page 372]. A modern paraphrase of POPA by Bishop [22, page 43] is the following:

A subject may not give rights it does not possess to another.

In other words, the granter of a right must possess the right.

In FSNSs, there is no explicit granting of rights. Authorization is the consequence of the social graph possessing a particular topology. Granting and revocation of access occur implicitly when befriending and defriending actions alter the topology of the social graph. We adapt POPA to the context of FSNSs, and devise below a formulation of POPA that prevents Sybil attacks:

Every action (e.g., befriending or defriending) that could cause an unprivileged subject to eventually gain access must be initiated by (i.e., have the consent of) a subject who already has access.

To see how POPA prevents Sybil attacks, note that both the attacker's original account and the new accounts she creates lack the privilege to access the target profile item. Actions initiated solely within this group of user accounts, according to POPA, could never cause any of them to eventually gain access. To gain access, they need the consent of users who already possess the required access. (This informal argument connecting POPA to Sybil attack prevention will be made formal in Section IV-C.)

\section{Our Approach}

Promising as it is, the above statement of POPA leads to two intellectual challenges. First, what precisely do we mean 
when we say "an action causes a subject to eventually gain access." How does one formalize such a notion of causality, which is foundational to POPA itself? Second, how does one design or configure a FSNS to ensure it realizes POPA? To the very least, is there a way for us to validate that an FSNS is POPA compliant? The main contributions of this work are concrete means to address these two challenges. We will capture causality by a noninterference-style formulation of POPA, and devise a static analysis to check if a given FSNS design or configuration is POPA compliant. Specifically, our plan is the following:

1) We will formalize POPA as a noninterference-style property [1], [2] of the set of execution traces of a given FSNS (i.e., a run-time property).

2) We will identify a structural property that can be imposed on the policy vocabulary of the FSNS to guarantee POPA (i.e., a static property).

3) We will prove a soundness result: If the policy vocabulary satisfies the static property in Step 2, then the set of execution traces generated by the FSNS will satisfy the run-time property of Step 1.

\section{MODELLING FSNSS}

This work is built on a formal model of FSNSs and a theory of FSNS policies that were previously proposed in [7], [8]. In the following, we review, adapt and extend these results.

\section{A. Basic Notations}

We write $\mathbb{B}$ to denote the set $\{0,1\}$ of Boolean values. Given a set $S, 2^{S}$ is the power set of $S,[S]^{k}$ is the set of all size$k$ subsets of $S$, and, when $S$ is finite and non-empty, $\mathcal{G}(S)$ is the set of all simple graphs with $S$ as the vertex set (i.e., $\left.\mathcal{G}(S)=\left\{\langle S, E\rangle \mid E \subseteq[S]^{2}\right\}\right)$. Given $e \in[V(G)]^{2}$, we write $G+e$ to denote the graph $\langle V(G), E(G) \cup\{e\}\rangle$. We write $G_{1} \subseteq G_{2}$ whenever graph $G_{1}$ is a subgraph of graph $G_{2}$ (i.e., $V\left(G_{1}\right) \subseteq V\left(G_{2}\right)$ and $\left.E\left(G_{1}\right) \subseteq E\left(G_{2}\right)\right)$. We also write $G_{1} \cup$ $G_{2}$ to denote the graph $\left\langle V\left(G_{1}\right) \cup V\left(G_{2}\right), E\left(G_{1}\right) \cup E\left(G_{2}\right)\right\rangle$. Fixing a vertex set $V$, we write $G^{\emptyset}$ for the empty graph $\langle V, \emptyset\rangle$. A vertex set $V$ is a vertex cover of an edge set $E$ iff $V \cap e \neq \emptyset$ for every $e \in E$.

\section{B. A Model of FSNSs}

1) Policies: Fixing a set $S u b$ of users, we model a policy as a predicate (i.e., a Boolean function) with the signature $S u b \times S u b \times \mathcal{G}(S u b) \rightarrow \mathbb{B}$. Given a profile owner $u \in S u b$, a profile accessor $v \in S u b$, and the current social graph $G \in$ $\mathcal{G}(S u b)$, a policy predicate returns a Boolean value indicating if access should be authorized.

Example 2. The policy predicates dist $_{k}, c f_{k}$ and clique ${ }_{k}$ are defined as follows: $\operatorname{dist}_{k}(u, v, G)$ holds whenever there is a path of length $k$ or less between $u$ and $v$ in $G ; c f_{k}(u, v, G)$ holds iff either $\operatorname{dist}_{1}(u, v, G)$ or else $u$ and $v$ share at least $k$ distinct common friends in $G$; clique $_{k}(u, v, G)$ holds iff either $u=v$ or else $u$ and $v$ belong to a common clique of size $k$ in $G$. We write me, friend and fof as shorthands for dist $t_{0}$, dist $t_{1}$ and dist $t_{2}$ respectively. We also write $\top$ and $\perp$ to denote the constant predicates that always return true and false respectively.

We write $\mathcal{P} \mathcal{P}(S u b)$ to denote the set of policy predicates sharing the signature $S u b \times S u b \times \mathcal{G}(S u b) \rightarrow \mathbb{B}$.

2) Systems: An FSNS (or simply a system) $N$ is a triple $\langle S u b, P V, P o l\rangle$. The first component, $S u b$, is a finite set of users ${ }^{1}$. The second component, $P V$, called the policy vocabulary, specifies the set of legitimate policy predicates supported by the FSNS. Let $\mathcal{P} \mathcal{T}$ be the set $\{$ sch, tra, acc $\}$ of identifiers denoting the three types of policies (i.e., search, traversal and access). $P V: \mathcal{P} \mathcal{T} \rightarrow 2^{\mathcal{P P}(S u b)}$ is a family of policy sets indexed by the three policy types. Specifically, $P V$ (acc) is the set of policy predicates that a user can legitimately use as an access policy, and the interpretation of $P V$ (sch) and $P V$ (tra) is similar. Lastly, Pol, representing the privacy settings of the users, is a function with signature $S u b \times \mathcal{P} \mathcal{T} \rightarrow \mathcal{P} \mathcal{P}(S u b)$, such that $\operatorname{Pol}(u, t) \in P V(t)$. That is, $\operatorname{Pol}(u$, sch $), \operatorname{Pol}(u$, tra $)$ and $\operatorname{Pol}(u$, acc $)$ are respectively the search, traversal and access policy of user $u$. In this work, we do not consider acquaintance policies.

3) States: A state of an FSNS is simply a social graph. We write $\mathcal{S}_{N}$ to denote the set $\mathcal{G}(S u b)$ of all states of $N$. In this work, we do not consider the management aspect of an FSNS, and thus we do not model user-initiated change of policies.

4) Queries: We model access as queries over states. Specifically, we write the sequent $G \vdash_{N} q$ whenever the social graph $G$ satisfies the query $q$. There are two types of query:

$$
q::=v \text { reads } u \mid v \text { finds } u
$$

The sequent " $G \vdash_{N} v$ finds $u$ " holds whenever accessor $v$ can reach the search listing of owner $u$ in state $G$ of system $N$. The sequent " $G \vdash_{N} v$ reads $u$ " holds whenever accessor $v$ is authorized to access the profile of owner $u$ in state $G$. Formally, the sequents are defined as follows:

$$
\begin{aligned}
& \frac{N=\left\langle_{-},{ }_{-}, \operatorname{Pol}\right\rangle \quad \operatorname{Pol}(u, \mathrm{sch})(u, v, G)}{G \vdash_{N} v \text { finds } u}(\mathrm{~F}-\mathrm{SCH}) \\
& \frac{u=v}{G \vdash_{N} v \text { finds } u} \\
& \frac{\{u, v\} \in E(G)}{G \vdash_{N} v \text { finds } u} \\
& G \vdash_{N} v \text { finds } u^{\prime} \quad\left\{u^{\prime}, u\right\} \in E(G) \\
& \frac{N=\left\langle{ }_{-}, \operatorname{Pol}_{\rangle} \quad \operatorname{Pol}\left(u^{\prime}, \text { tra }\right)\left(u^{\prime}, v, G\right)\right.}{G \vdash_{N} v \text { finds } u}(\text { F-TRv }) \\
& G \vdash_{N} v \text { finds } u \\
& \frac{N=\left\langle_{-},{ }_{-}, \operatorname{Pol}\right\rangle \quad \operatorname{Pol}(u, \text { acc })(u, v, G)}{G \vdash_{N} v \text { reads } u}(\mathrm{R}-\mathrm{ACC})
\end{aligned}
$$

\footnotetext{
${ }^{1}$ Rather than modelling a system with an evolving number of users and thus complicating the model unnecessarily, we fix the number of users in a system. User addition could be seen as certain dormant users becoming active. User
} removal can be modelled in the opposite way. 
In each of the queries " $v$ reads $u$ " and " $v$ finds $u$ ", we call $v$ the accessor of the query. Given query $q$, we write $q(v)$ to denote the query obtained from $q$ by substituting $v$ for the accessor of $q$. For example, if $q$ is " $v$ reads $u$ ", then $q\left(v^{\prime}\right)$ is the query " $v$ ' reads $u$ ". Accessor substitution constructs a query that exercises the same right as the original query, but now the right is exercised by a different user.

5) Transitions: There are only two types of transitions: befriending and defriending, identified respectively by transition identifiers of the forms $e$ and $\bar{e}$, where $e$ is the edge in the social graph affected by the transition. A befriending transition, identified by $e$, requires the consent of both parties involved. A defriending transition, identified by $\bar{e}$, can be initiated unilaterally by either end of the edge $e$. Let $\mathcal{T}_{N}$ be the set of all transition identifiers.

The transition relation $\cdot \stackrel{\cdot}{\longrightarrow}_{N}: \mathcal{S}_{N} \times \mathcal{T}_{N} \times \mathcal{S}_{N}$ is defined as follows:

$$
\begin{array}{ll}
G \stackrel{e}{\longrightarrow}{ }_{N} G+e & \text { if } e \notin E(G) \\
G \stackrel{\bar{e}}{\longrightarrow} N G-e & \text { if } e \in E(G) .
\end{array}
$$

Notice that befriending is unmediated, meaning that everyone can befriend everyone else. It is no longer necessary to reach the search listing of the target user and seek authorization by her acquaintance policy in order to initiate befriending (see Section II-A). We call this simplifying assumption Unmediated Befriending.

6) Traces: A finite sequence from $\left(\mathcal{T}_{N}\right)^{*}$ is called a trace. We generalize the transition relation to traces. Specifically, we write $G \stackrel{\epsilon}{\longrightarrow} N G$, and, given $\tau, \pi \in\left(\mathcal{T}_{N}\right)^{*}$, we write $G \stackrel{\tau \cdot \pi}{\longrightarrow}{ }_{N} G^{\prime}$ whenever $G \stackrel{\tau}{\longrightarrow}{ }_{N} G^{\prime \prime}$ and $G^{\prime \prime} \stackrel{\pi}{\longrightarrow}{ }_{N} G^{\prime}$ for some social graph $G^{\prime \prime}$. A trace $\tau$ is feasible in $G$ iff $G \stackrel{\tau}{\longrightarrow}{ }_{N} G^{\prime}$ for some $G^{\prime}$. This feasible trace $\tau$ establishes $q$ in $G$ whenever " $G \vdash_{N} q$ " holds but " $G \vdash_{N} q$ " does not.

7) Discussion: We have significantly simplified the FSNS model previously proposed in [7], [8]. The simplifications, which are summarized below, have been instrumental in making the results in this work feasible.

- A policy predicate is parameterized only by the owner, the accessor and the social graph. The model in [7], [8] allows a limited form of history information to affect authorization decisions.

- All policies (i.e., traversal, search, and access) are fixed: i.e., the privacy settings of users remain constant at runtime. The model in [7], [8] captures the management aspect of the access control system, and thus the change of policies at run time.

- We use a single access policy to protect the entire profile, thereby removing the need to track a different access policy for each profile item. Extending the theory to handing multiple profile items is a straightforward exercise [7], [8].

- The only user actions in the model are befriending and defriending. The model in [7], [8] permits a more complex consenting protocol. Furthermore, the two actions are atomic. Specifically, we model the multi-step protocol of obtaining consent as a single transition in our model.

- Befriending is unmediated: everyone can befriend everyone else.

\section{A Theory of Policies}

Recall that a policy predicate is a member of $\mathcal{P} \mathcal{P}(S u b)$, the set of predicates with the signature $S u b \times S u b \times \mathcal{G}(S u b) \rightarrow$ $\mathbb{B}$. Fong et al. [7], [8] provided characterization of certain families of policy predicates. These results are reviewed here. We begin with the notion of birooted graphs, on which these characterization results are based.

Definition 3. A birooted graph $G_{(u, v)}$ is a triple $\langle G, u, v\rangle$ such that $G$ is a simple graph and $u, v \in V(G)$. The roots $u$ and $v$ need not be distinct. We write $\mathcal{B}(S)=\left\{G_{(u, v)} \mid u, v \in\right.$ $S, G \in \mathcal{G}(S)\}$ to denote the set of all birooted graphs with vertex set $S$.

$G^{\prime}{ }_{(u, v)}$ is a (birooted) subgraph of $G_{(u, v)}$, written as $G^{\prime}{ }_{(u, v)} \subseteq G_{(u, v)}$, iff $G^{\prime}$ is a subgraph of $G$. (Note the matching roots.) We say that $G_{(u, v)}$ and $G^{\prime}{ }_{\left(u^{\prime}, v^{\prime}\right)}$ are isomorphic iff there exists a graph isomorphism $\phi: V(G) \rightarrow V\left(G^{\prime}\right)$ between $G$ and $G^{\prime}$, such that $\phi(u)=u^{\prime}$ and $\phi(v)=v^{\prime}$. In this case we write $G_{(u, v)} \cong G^{\prime}{ }_{\left(u^{\prime}, v^{\prime}\right)}$. We also write $G^{\prime}{ }_{\left(u^{\prime}, v^{\prime}\right)} \lesssim G_{(u, v)}$ whenever there is a birooted graph $G^{\prime \prime}{ }_{(u, v)}$ such that $G^{\prime \prime}{ }_{(u, v)} \subseteq G_{(u, v)}$ and $G^{\prime}{ }_{\left(u^{\prime}, v^{\prime}\right)} \cong G^{\prime \prime}{ }_{(u, v)}$.

Other than the above cases, when we apply graph-theoretic languages to birooted graphs, we are essentially referring to the underlying simple graphs.

Not all policies are equal. Some exhibit characteristics that are security relevant.

Definition 4. A policy $P$ is monotonic iff $G_{(u, v)} \subseteq G^{\prime}{ }_{(u, v)}$ implies $P(u, v, G) \Rightarrow P\left(u, v, G^{\prime}\right)$. Policy $P$ is topology-based iff $G_{(u, v)} \cong G^{\prime}\left(u^{\prime}, v^{\prime}\right)$ implies $P(u, v, G)=P\left(u^{\prime}, v^{\prime}, G^{\prime}\right)$. Policy $P$ is positive iff it is both monotonic and topologybased.

With a monotonic policy, adding edges to the social graph never diminishes accessibility. Similarly, removing edges from the social graph never increases accessibility. With a topologybased policy, topologically equivalent access scenarios, represented by isomorphic birooted graphs, produce identical authorization decisions. In short, the individual identity of the users involved are not considered in the authorization decision. A topology-based policy therefore expresses a graph-theoretic relation between two vertices.

Example 5. All policy predicates in Example 2 are monotonic, topology-based and positive.

There are alternative ways to characterize monotonic and positive policies.

Definition 6. Let $\mathcal{B} \in \mathcal{B}(S u b)$ be a set of birooted graphs. The policy monotonically induced by $\mathcal{B}$ is the predicate $P_{\mathcal{B}}^{M}$ for which

$$
P_{\mathcal{B}}^{M}(u, v, G) \text { iff } \exists G^{\prime}{ }_{(u, v)} \in \mathcal{B} \cdot G^{\prime}{ }_{(u, v)} \subseteq G_{(u, v)}
$$


The policy positively induced by $\mathcal{B}$ is the predicate $P_{\mathcal{B}}^{+}$for which

$$
P_{\mathcal{B}}^{+}(u, v, G) \text { iff } \exists G^{\prime}{ }_{\left(u^{\prime}, v^{\prime}\right)} \in \mathcal{B} \cdot G^{\prime}{ }_{\left(u^{\prime}, v^{\prime}\right)} \lesssim G_{(u, v)}
$$

The following theorem provides an alternative characterization to policies that are respectively monotonic and positive.

Theorem 7. Every monotonic (resp. positive) policy predicate is monotonically (resp. positively) induced by a set of birooted graphs. The minimal set of birooted graphs to monotonically (resp. positively) induce a given predicate $P$ is defined to be the set $\mathcal{B}$ for which there exists no proper subset of $\mathcal{B}$ that also monotonically (resp. positively) induces $P$. This minimal set does not contain a pair of distinct birooted graphs $G_{(u, v)}$ and $G^{\prime}{ }_{\left(u^{\prime}, v^{\prime}\right)}$ such that $G_{(u, v)} \subseteq G^{\prime}{ }_{\left(u^{\prime}, v^{\prime}\right)}$ (resp. $G_{(u, v)} \lesssim$ $\left.G^{\prime}{ }_{\left(u^{\prime}, v^{\prime}\right)}\right)$. Such a minimal set always exists, and is unique (resp. unique up to birooted graph isomorphism).

The theorem has two versions, one regarding monotonic policy predicates, the other regarding positive predicates. The positive version of the theorem has been previously stated and proved in [8]. The monotonic version is new, and its proof follows that of the positive version closely.

Example 8. Let $S u b=\left\{u_{0}, u_{1}, \ldots, u_{n}\right\}$, where $n \geq 2$. Consider the sets $\mathcal{B}_{\text {dist }_{k}}, \mathcal{B}_{c f_{k}}, \mathcal{B}_{\perp}$ and $\mathcal{B}_{\top}$ to positively induce dist $_{k}, \mathrm{cf}_{k}, \perp$ and $\top$ respectively (assuming $1 \leq k \leq n$ ).

1) $\mathcal{B}_{\text {dist }_{k}}=\left\{G_{\left(u_{0}, u_{i}\right)} \mid 0 \leq i \leq k\right\}$, where the graph $G^{i}$ contains exactly the edges that form the path $u_{0} u_{1} \ldots u_{i}$.

2) $\mathcal{B}_{c f_{k}}=\left\{G_{\left(u_{0}, u_{0}\right)}^{\emptyset}, G^{b}{ }_{\left(u_{0}, u_{1}\right)}, G^{\sharp}{ }_{\left(u_{0}, u_{k+1}\right)}\right\}$, where $E\left(G^{b}\right)=\left\{\left\{u_{0}, u_{1}\right\}\right\}, E\left(G^{\sharp}\right)=\left\{\left\{u_{0}, u_{i}\right\} \mid 1 \leq i \leq\right.$ $k\} \cup\left\{\left\{u_{i}, u_{k+1}\right\} \mid 1 \leq i \leq k\right\}$.

3) $\mathcal{B}_{\perp}=\emptyset$.

4) $\mathcal{B}_{\top}=\left\{G_{\left(u_{0}, u_{0}\right)}^{\emptyset}, G_{\left(u_{0}, u_{1}\right)}^{\emptyset}\right\}$

We define policy combinators that allow us to create complex policies from primitive ones (the combinators $\vee$ and $\wedge$ were defined in [7], [8]; the combinators $\circ$ and $\cdot(\cdot \cdot)$ are new).

Definition 9. Given policy predicates $P, P_{1}$ and $P_{2}$, and vertex $u_{0}$, we define the composite predicates $P_{1} \vee P_{2}, P_{1} \wedge P_{2}$, $P_{1} \circ P_{2}$ and $P\left(u_{0}\right)$ as follows:

$$
\begin{aligned}
\left(P_{1} \vee P_{2}\right)(u, v, G) & =P_{1}(u, v, G) \vee P_{2}(u, v, G) \\
\left(P_{1} \wedge P_{2}\right)(u, v, G) & =P_{1}(u, v, G) \wedge P_{2}(u, v, G) \\
\left(P_{1} \circ P_{2}\right)(u, v, G) & =\left(\exists u^{\prime} \in S u b . P_{1}\left(u, u^{\prime}, G\right) \wedge P_{2}\left(u^{\prime}, v, G\right)\right) \\
P\left(u_{0}\right)(u, v, G) & =\left(u=u_{0}\right) \wedge P(u, v, G)
\end{aligned}
$$

The following proposition is new.

Proposition 10. Suppose policy predicates $P, P_{1}$ and $P_{2}$ are monotonically induced by $\mathcal{B}, \mathcal{B}_{1}$ and $\mathcal{B}_{2}$, and $u_{0}$ is a vertex. Then $P_{1} \vee P_{2}, P_{1} \wedge P_{2}, P_{1} \circ P_{2}$ and $P\left(u_{0}\right)$ are monotonically induced by the following sets respectively:

$$
\begin{gathered}
\mathcal{B}_{1} \cup \mathcal{B}_{2} \\
\left\{\left(G_{1} \cup G_{2}\right)_{(u, v)} \mid G_{1(u, v)} \in \mathcal{B}_{1} \wedge G_{2(u, v)} \in \mathcal{B}_{2}\right\} \\
\left\{\left(G_{1} \cup G_{2}\right)_{(u, v)} \mid\right. \\
\left.\exists u^{\prime} \in S u b . G_{1\left(u, u^{\prime}\right)} \in \mathcal{B}_{1} \wedge G_{2\left(u^{\prime}, v\right)} \in \mathcal{B}_{2}\right\} \\
\left\{G_{\left(u_{0}, v\right)} \mid G_{\left(u_{0}, v\right)} \in \mathcal{B}\right\}
\end{gathered}
$$

While the proof of the proposition is elementary, there are two important corollaries that we will rely on in the sequel. The first and obvious one is that monotonicity is preserved by the four combinators. The second corollary is not as direct. Suppose $\mathcal{B}, \mathcal{B}_{1}$ and $\mathcal{B}_{2}$ are not just any sets to monotonically induce $P, P_{1}$ and $P_{2}$, but the minimal ones to do so. Then, by the proposition above, the minimal sets to monotonically induce $P_{1} \vee P_{2}, P_{1} \wedge P_{2}, P_{1} \circ P_{2}$ and $P\left(u_{0}\right)$ are respectively subsets of the four sets identified above. This, in turn, means that we know the "shape" of the members of the minimal sets to monotonically induce the four composite policies. For example, every member of the minimal set to monotonically induce $P_{1} \wedge P_{2}$ must be of the form $\left(G_{1} \cup G_{2}\right)_{(u, v)}$, where $G_{1(u, v)}$ and $G_{2(u, v)}$ are respectively members of $\mathcal{B}_{1}$ and $\mathcal{B}_{2}$.

\section{POPA FORMALIZED}

We follow a two-step plan to devise a formal statement of POPA. First, we will define the notion of "reduction", which allows us to "blame" certain transitions in a trace as the causal enablers of access granting. Second, we will define the notion of "rationalization", which allows us to "explain", after the fact, how authority is conferred among the causal enablers identified above, ultimately leading to the granting of access. POPA is then expressed as a statement asserting that it is possible to explain authority conferral among the causal enablers in such a way that only privileged users may confer privilege to unprivileged users. Lastly, we demonstrate that this definition of POPA eliminates Sybil attacks.

In this section and in the rest of this paper, we will make the following assumption.

Assumption 11 (Monotonicity). All policy predicates considered are monotonic.

In particular, this implies that each of $P V$ (sch), $P V$ (tra) and $P V($ acc $)$ contains only monotonic policies. Proposition 10 guarantees that policies constructed out of the policy vocabulary using the policy combinators will remain monotonic.

\section{A. Reduction}

In a trace that establishes access, not every transition in the trace is causally relevant to the eventual granting of access. We want to be able to identify those that are relevant, and impose on them constraints regarding conferral of privileges. To this end, we define a notion of reduction in the style of noninterference. 
Definition 12. Suppose $\tau$ establishes $q$ in $G$. A subsequence $e^{2}$ $\pi$ of $\tau$ is a $(G, q)$-reduction (or simply a reduction) of $\tau$ iff $\pi$ also establishes $q$ in $G$. Trace $\tau$ is minimally $(G, q)$ reduced (or simply minimally reduced) iff $\tau$ is the only $(G, q)$ reduction of itself. We call $\pi$ a minimal $(G, q)$-reduction (or simply minimal reduction) of $\tau$ if $\pi$ is a $(G, q)$-reduction of $\tau$ that is minimally $(G, q)$-reduced.

By definition, reducibility is reflexive, transitive and antisymmetric. That is, reduction imposes a partial ordering over the set of traces.

Intuitively, a reduction $\pi$ is a "simplified" version of $\tau$, with the transitions inessential for establishing $q$ removed. A minimal reduction of $\tau$ is a reduction $\pi$ that does not contain another reduction as a proper subsequence. That is, it contains the transitions that are essential for establishing $q$. These transitions are considered to have "caused" $q$ to be established. Note that a feasible trace may have more than one minimal reductions. Under Assumption 11 (Monotonicity), the following observation can be made.

Proposition 13. Every transition of a minimally reduced trace is a befriending transition (i.e., with a transition identifier e).

In short, defriending is never necessary for establishing access when all policies are monotonic. By the observation above, a minimal reduction of length $n$ has the form $e_{0} e_{1} \ldots e_{n-1}$.

Under the assumption of Unmediated Befriending, every user can befriend every other user at will (so long as consent is granted by the other party). A consequence is the following observation, which can be proved easily by contradiction.

Proposition 14. Every permutation of a minimally reduced trace is also feasible and minimally reduced.

\section{B. Rationalization}

We define what it means for a minimally reduced trace to properly observe the requirement of POPA.

Definition 15. Suppose $\tau=e_{0} e_{1} \ldots e_{n-1}$ is a minimally $(G, q)$-reduced trace for system $N$. Then $\tau$ is admissible whenever:

1) $G=G_{0}{\stackrel{e_{0}}{\longrightarrow}}_{N} G_{1}{\stackrel{e_{1}}{\longrightarrow}}_{N} \ldots G_{n-1} \stackrel{e_{n-1}}{\longrightarrow} G_{n}=$ $G^{\prime \prime}$ for some social graph $G^{\prime \prime} \in \mathcal{S}_{N}$, and

2) there is a sequence of vertices $v_{0}, v_{1}, \ldots, v_{n-1}$ such that $v_{i} \in e_{i}$ and $G_{i} \vdash_{N} q\left(v_{i}\right)$ for $1 \leq i<n$. Note that the sequence may contain repeated vertices.

A trace $\pi$ satisfying requirements 1 and 2 above establishes $q$ in a principled manner. Since befriending transition $e_{i}$ furthers the establishment of $q$, we interpret the addition of $e_{i}$ as a privilege conferral event. In accordance with the intuition of POPA, such a privilege conferral event must be initiated by someone who already possesses the conferred privilege. As a befriending action $e_{i}$ is jointly initiated by both ends

\footnotetext{
${ }^{2}$ E.g., $a c b$ is a subsequence of $a b c b a$. As special cases, every sequence is its own subsequence, and the empty sequence is a subsequence of every sequence.
}

of the edge, we therefore require that one of its ends (i.e., $v_{i} \in e_{i}$ ) to already possess the conferred privilege. That is, we require that, in the state $G_{i}$ in which the privilege is conferred, the initiator above already possess the privilege: i.e., $G_{i} \vdash_{N} q\left(v_{i}\right)$. The sequence $\pi$ therefore honors POPA in the way it incrementally establishes $q$.

According to Proposition 14, every permutation of a minimally reduced trace is also minimally reduced, and thus every such permutation also establishes the query in question. Some of these permutations may be admissible.

Definition 16. Suppose $\tau$ is a minimally $(G, q)$-reduced trace. Then a permutation ${ }^{3} \pi$ of $\tau$ is a rationalization of $\tau$ whenever $\pi$ is admissible.

If a minimally reduced trace has a rationalization, it means that we can explain, after the fact, by rearranging the transitions in the trace, how privilege could have been incrementally conferred in accordance to POPA. Not every minimally reduced trace has a rationalization.

Definition 17. A system $N$ is POPA-compliant iff, for every $G \in \mathcal{S}_{N}$, every query $q$ of the form " $v$ reads $u$ ", and every $\tau \in\left(\mathcal{T}_{N}\right)^{*}$ that establishes $q$ in $G$, it is the case that every minimal $(G, q)$-reduction of $\tau$ has a rationalization.

Note that POPA compliance is not a property of individual traces, but rather an assertion regarding the correlation of traces generated by the system (i.e., "if such a trace is feasible then such other traces are also feasible" [23]). It employs a noninterference-style condition [1], [2] to filter away inessential transitions in a given trace, and then assert the existence of an explanation of how individual transitions could have been arranged such that at least one initiator of each transition is authorized for access when the transition occurs.

\section{Sybil Attack Prevention}

POPA compliance is a strong condition: it requires every conferral of privilege to be initiated by a user possessing the conferred privilege. An interesting question is whether such a strong requirement is actually necessary for preventing Sybil attacks. Intuitively, to prevent Sybil attacks, all we need is that a group of unprivileged users cannot collude to gain privilege (Section II-B). That is, the establishment of privilege requires the cooperation of at least one privileged user. In the following, we demonstrate that the two conditions are in fact equivalent, and thus POPA compliance is not only sufficient but also necessary for preventing Sybil attacks.

The following scenario constitutes a Sybil attack in system $N=\langle S u b, P V, P o l\rangle$. Suppose $q$ is a query of the form "v reads $u$ ", $G \in \mathcal{S}_{N}$ is a state in which $G \vdash_{N} q$ does not hold, and $\tau \in\left(\mathcal{T}_{N}\right)^{*}$ is a trace that establishes $q$ in $G$. Under Assumption 11, defriending transitions in $\tau$ are not significant. We focus on the befriending transitions. A Sybil

\footnotetext{
${ }^{3}$ E.g., bcad is a permutation of $a b c d$. As a special case, every sequence is a permutation of itself.
} 
attack has occurred if, for every befriending transition $e$ in $\tau$, and every initiator $v^{\prime} \in e, G \vdash_{N} q\left(v^{\prime}\right)$ does not hold. That is, none of those contributing to the establishment of $q$ possesses the established privilege in the initial state $G$. The following definition captures the condition under which a system is not susceptible to Sybil attacks.

Definition 18. A system $N=\langle S u b, P V, P o l\rangle$ is Sybil free iff the following holds: For every query $q$ of the form " $v$ reads $u$," for every state $G \in \mathcal{S}_{N}$ in which $G \vdash_{N} q$ does not hold, and for every trace $\tau \in\left(\mathcal{T}_{N}\right)^{*}$ that establishes $q$ in $G$, there exists a befriending transition $e$ in $\tau$, with an initiator $v^{\prime} \in e$, such that $G \vdash_{N} q\left(v^{\prime}\right)$.

Intuitively, the condition above requires that every trace that establishes a privilege must contain a transition initiated by a user who possesses the privilege before the trace is executed. That is, it is impossible for a group of users who do not possess a privilege to collude and cause one of them to gain privilege.

Theorem 19. A system is Sybil free iff it is POPA compliant.

This result is surprising, because POPA compliance is apparently stronger than Sybil freedom. Yet it turns out that the two are equivalent. This means POPA compliance is the not only sufficient for preventing Sybil attacks, but also necessary. As such POPA compliance gives us a tight condition for Sybil attack prevention.

Proof: Let $N=\langle S u b, P V, P o l\rangle$ be the system in question. We begin with the "if" direction. Suppose $N$ is POPA compliant. Consider a query $q$ of the form " $v$ reads $u$ ", a state $G \in \mathcal{S}_{N}$ such that $G \vdash_{N} q$ does not hold, and a trace $\tau \in\left(\mathcal{T}_{N}\right)^{*}$ that establishes $q$ in $G$. POPA compliance (Definition 17) guarantees that there exists a rationalization $\pi$ for every minimal $(G, q)$-reduction of $\tau$. We know that $\pi$ contains at least one transition, and that every transition in $\pi$ is a befriending transition (Proposition 13). Let $e$ be the first transition in $\pi$. Now, $e$ also occurs in $\tau$, and by Definition 15 we know $G \vdash_{N} q\left(v^{\prime}\right)$ for some initiator $v^{\prime}$ of $e$. Therefore, $N$ is Sybil free.

We now prove the "only-if" direction. Suppose $N$ is Sybil free. Consider a query $q$ of the form " $v$ reads $u$ ", states $G, G^{\prime} \in \mathcal{S}_{N}$, and a trace $\tau \in\left(\mathcal{T}_{N}\right)^{*}$ such that $G \stackrel{\tau}{\longrightarrow}{ }_{N} G^{\prime}$. Suppose further $G \vdash_{N} q$ does not hold but $G^{\prime} \vdash_{N} q$ does. Let $\pi$ be any minimal $(G, q)$-reduction of $\tau$. Our goal is to construct a rationalization $\pi^{\prime}$ for $\pi$. We describe in the following an inductive procedure for constructing $\pi^{\prime}$.

We begin with the base case, in which $\pi$ contains only one transition $e_{0}$. Sybil freedom guarantees that one of the initiator $v_{0} \in e_{0}$ is such that $G \vdash_{N} q\left(v_{0}\right)$. Thus we can simply take $\pi^{\prime}=\pi$ to satisfy the requirement of admissibility (Definition $15)$.

In the induction step, consider $\pi=e_{0} e_{1} \ldots e_{n-1}$, for some $n>1$, such that:

$$
G=G_{0}{\stackrel{e_{0}}{\longrightarrow}}_{N} G_{1} \stackrel{e_{1}}{\longrightarrow}{ }_{N} \ldots G_{n-1} \stackrel{e_{n-1}}{\longrightarrow}{ }_{N} G_{n}=G^{\prime \prime}
$$

for some social graph $G^{\prime \prime} \in \mathcal{S}_{N}$. Sybil freedom guarantees that there exists a transition $e_{i}, 0 \leq i<n$, such that one of its initiators $v_{i} \in e_{i}$ satisfies $G \vdash_{N} q\left(v_{i}\right)$. Let $\pi=\pi_{1} e_{i} \pi_{2}$ for some $\pi_{1}, \pi_{2} \in\left(\mathcal{T}_{N}\right)^{*}$. Now consider the trace $\pi^{\sharp}=e_{i} \pi_{1} \pi_{2}$ obtained by moving $e_{i}$ to the front of the sequence, leaving all the other transitions as is. By Proposition 14 the trace $\pi^{\sharp}$ is also a minimal $(G, q)$-reduction. Suppose

$$
G \stackrel{e_{i}}{\longrightarrow}{ }_{N} G^{\sharp} \stackrel{\pi_{1} \pi_{2}}{\longrightarrow}{ }_{N} G^{\prime \prime}
$$

We know that $G^{\sharp} \vdash_{N} q$ does not hold, or else $\pi^{\sharp}$ would not have been a minimally reduced trace. This means $\pi_{1} \pi_{2}$ is also a minimal $(G, q)$-reduction. By induction, we can construct a rationalization $\pi^{\mathrm{b}}$ of $\pi_{1} \pi_{2}$. We take $\pi^{\prime}=e_{i} \pi^{\mathrm{b}}$ to be the desired rationalization of $\pi$.

\section{A Static Analysis}

POPA compliance is a property of the set of feasible traces generated by an FSNS. As such it is a dynamic property. Our goal in this section is to devise a static analysis so that one can ascertain that a given FSNS is POPA compliant without having to examine all feasible traces of the system. To this end we will formulate a static property regarding the policy vocabulary of an FSNS. We will establish a soundness theorem, which asserts that, if an FSNS has a policy vocabulary conforming to the said static property, then the system is POPA compliant. One does not generally expect such a static property to be complete: i.e., every POPA-compliant system possesses such a static property. To our surprise, the static property we are to formulate is indeed complete.

\section{A. Assumptions}

In this section, we target our analysis on the last line of defence of an FSNS: the access policies. This means we do not consider Stage-I Authorization to be a proper component of access authorization, but rather, merely as an idiosyncratic means for, say, protecting the privacy of the users' friend lists. This narrowing of focus is captured in the following assumption.

Assumption 20 (Unmediated Access). $P V(s c h)=\{\top\}$.

With this assumption, the only search policy that can be adopted will be $T$. That is, global name search will always succeed. The effect is that profile access is now guarded only by the access policy, but not by the reachability of the owner's search listing, because the search listing is now rendered always reachable. Essentially, we are replacing (R-ACC) by the following inference rule:

$$
\frac{N=\left\langle{ }_{-}, \operatorname{Pol}\right\rangle \quad \operatorname{Pol}(u, \operatorname{acc})(u, v, G)}{G \vdash_{N} v \text { reads } u}\left({\left.\mathrm{R}-\mathrm{ACC}^{-}\right)}^{-}\right.
$$

The significance of this assumption can be understood in terms of constraining the scope of "blaming". Recall that POPA compliance is defined in terms of reduction, which is a means for discarding from consideration the set of transitions that do not contribute to the establishment of access. By removing from (R-ACC) the precondition of reaching the owner's search listing, we are essentially refusing to "blame" 
a transition if it contributes only to making the owner's search listing reachable, but not to satisfying the owner's access policy.

\section{B. The Substructure Property}

We state the Substructure Property, which can be employed as the basis for a static analysis that verifies if a given FSNS is POPA compliant.

Definition 21. Suppose $N=\langle S u b, P V$, Pol $\rangle$ is an FSNS, and $\mathcal{B}$ is the minimal set of birooted graphs to monotonically induce a predicate $P \in \mathcal{P} \mathcal{P}(S u b)$. Then $P$ has the Substructure Property (SP) at $u \in S u b$ iff the following holds:

For every $G_{(u, v)} \in \mathcal{B}$, there exists a non-empty, finite sequence of distinct vertices $v_{0}, v_{1}, \ldots, v_{n}=v$ from $S u b$, and a corresponding sequence of graphs $G_{0}$, $G_{1}, G_{2}, \ldots, G_{n}=G$ from $\mathcal{G}(S u b)$, such that, for $0 \leq i \leq n$, we have: (a) $G_{i} \subseteq G$, (b) $G_{i\left(u, v_{i}\right)}$ is a member of $\mathcal{B}$, and (c) the vertex set $V_{i}$ is a vertex cover for $E\left(G_{i}\right)$, where $V_{0}=\emptyset$, and $V_{i}=$ $\left\{v_{0}, \ldots, v_{i-1}\right\}$ for $1 \leq i \leq n$.

We say that $P$ has $S P$ iff $P$ has $S P$ at every $u \in S u b$.

Definition 22. An FSNS $N=\langle S u b, P V, P o l\rangle$ has SP iff, for every $u \in S u b$, the policy predicate $\operatorname{Pol}(u, a c c)$ has $S P$ at $u$.

In the sequel, we will show that an FSNS that has SP is also POPA compliant, meaning that SP can serve as a static analysis for verifying POPA compliance. In practice, it would be inefficient to verify SP at every vertex, as the number of users can be very large. The following corollary prescribes a more tractable procedure for static analysis: one examines the policy vocabulary of the system rather than the individual privacy settings of users.

Corollary 23. An FSNS $N=\langle S u b, P V$, Pol $\rangle$ has $S P$ if every policy predicate in $P V($ acc $)$ has $S P$.

Note that the procedure above checks whether each access policy as a whole has SP, rather than checking if some access policy has SP at a specific vertex. Still, checking whether a monotonic predicate has SP can be tedious, as there are potentially many birooted graphs to consider in the minimal set to monotonically induce the predicate. Fortunately, if a policy is positive, then checking can be performed much more efficiently, as there are usually only a few birooted graphs to consider.

Corollary 24. Suppose $N=\langle S u b, P V$, Pol $\rangle$ is an FSNS, and $\mathcal{B}$ is the minimal set of birooted graphs to positively induce a predicate $P \in \mathcal{P} \mathcal{P}(S u b)$. Then $P$ has $S P$ iff the following holds:

For every $G_{(u, v)} \in \mathcal{B}$, there exists a non-empty, finite sequence of distinct vertices $v_{0}, v_{1}, \ldots, v_{n}=v$ from $S u b$, and a corresponding sequence of graphs $G_{0}$, $G_{1}, G_{2}, \ldots, G_{n}=G$ from $\mathcal{G}(S u b)$, such that, for $0 \leq i \leq n$, we have: (a) $G_{i} \subseteq G$, (b) $G_{i\left(u, v_{i}\right)}$ is isomorphic to a member of $\mathcal{B}$, and (c) the vertex set
$V_{i}$ is a vertex cover for $E\left(G_{i}\right)$, where $V_{0}=\emptyset$, and $V_{i}=\left\{v_{0}, \ldots, v_{i-1}\right\}$ for $1 \leq i \leq n$.

As we saw in Section III-C, birooted graphs could be used as a policy language for specifying the minimal set of "patterns" to positively induce a policy predicate. Corollary 24 is therefore the preferred form to use when the static analysis in Corollary 23 is to be performed. Note, however, the definition of SP is based on the more primitive notion of monotonic policies (instead of positive policies). As we shall see in the sequel, this minimalist approach will pay dividend when we work with policy predicates that are monotonic but not topology based.

Example 25. Let $S u b=\left\{u_{0}, u_{1}, \ldots, u_{n}\right\}$. The following are examples and counter-examples of SP.

1) The predicate dist $t_{k}$ has SP. To see this, recall from Example 8 that the minimal set to positively induce dist $k_{k}$ is $\mathcal{B}_{\text {dist }_{k}}=\left\{G_{\left(u_{0}, u_{i}\right)} \mid 0 \leq i \leq k\right\}$, where the graph $G^{i}$ contains exactly the edges that form the path $u_{0} u_{1} \ldots u_{i}$. For each $G^{i}{ }_{\left(u_{0}, u_{i}\right)}$, the vertex sequence $u_{0}, u_{1}, \ldots, u_{i}$ and graph sequence $G^{0}, G^{1}, \ldots, G^{i}$ satisfy the requirements of Corollary 24.

2) Predicate $\mathrm{Cf}_{k}$ has SP. Recall from Example 8 that the minimal set to positively induce $c f_{k}$ is $\mathcal{B}_{c f_{k}}=$ $\left\{G^{\emptyset}{ }_{\left(u_{0}, u_{0}\right)}, G^{b}{ }_{\left(u_{0}, u_{1}\right)}, G^{\sharp}{ }_{\left(u_{0}, u_{k+1}\right)}\right\}$, where $G^{b}$ and $G^{\sharp}$ are defined in Example 8. For $G^{\sharp}\left(u_{0}, u_{k+1}\right)$, the three requirements of Corollary 24 are satisfied by the vertex sequence $u_{0}, u_{1}, \ldots, u_{k+1}$ and the graph sequence $G^{0}, G^{1}, \ldots, G^{k+1}$, where $G^{0}=G^{\emptyset}, G^{k+1}=G^{\sharp}$, and $G^{i}=\left\langle S u b,\left\{\left\{u_{0}, u_{i}\right\}\right\}\right\rangle$ for $1 \leq i \leq k$.

3) The topology-based policies used in Facebook, including $\perp$, me, friend, fof and $\top$, have SP. To see this, note that me, friend and fof are but shorthands for dist ${ }_{k}$ policies, and $\perp$ and $T$ have SP by virtue of Proposition 27.1.

4) The policy predicate clique ${ }_{k}$ does not have SP at u for every $u \in S u b$, nor does the policy in Figure 1

The following observation is immediate.

Lemma 26. In Definition $21, G_{0}$ is an empty graph.

Additional facts about SP are the following:

Proposition 27. The following statements hold:

1) Both constant policies $\top$ and $\perp$ satisfy SP.

2) If $P_{1}$ and $P_{2}$ both have $S P\left(\right.$ at $u$ ), then $P_{1} \vee P_{2}$ also has $S P$ (at $u)$.

3) If $P$ has $S P$ (at $u$ ), then $P\left(u_{0}\right)$ has $S P$ (at $u$ ).

The proof of this proposition can be found in the companion technical report [24]. Statement 1 gives boundary examples of SP. Statements 2 and 3 offer ways to compose complex SPsatisfying policies from simpler ones. There are two versions to each of Statements 2 and 3: one with the "at $u$ " clauses, the other without. Note that SP is not preserved by $\wedge$ and $\circ$.

\section{Soundness and Completeness}

The connection between POPA as dynamic property and SP as a static analysis is captured in a pair of theorems: Soundness 
and Completeness. We begin with the main result of this work:

Theorem 28 (Soundness). If an FSNS has SP, then it is POPA compliant.

The soundness theorem allows us to examine the privacy settings of an FSNS, or more often its policy vocabulary (Corollary 23), to determine if the system is POPA compliant.

Proof: Suppose the FSNS $N=\langle S u b, P V, P o l\rangle$ has SP. We show that $N$ is POPA compliant.

Consider a social graph $G \in \mathcal{S}_{N}$, vertices $u, v \in S u b$, and a query $q$ of the form " $v$ reads $u$ ". Let $P=\operatorname{Pol}(u$, acc $)$. Let $\mathcal{B}$ be the minimal set of birooted graphs to monotonically induce $P$. Now, suppose $\neg P(u, v, G)$, and $G \stackrel{\tau}{\longrightarrow}{ }_{N} G^{\prime}$ for some trace $\tau \in\left(\mathcal{T}_{N}\right)^{*}$ and social graph $G^{\prime}$, such that $P\left(u, v, G^{\prime}\right)$. That is, $\tau$ establishes $q$ in $G$. We construct in the following the rationalization $\pi$ required by the definition of POPA compliance (Definition 17).

Because $P\left(u, v, G^{\prime}\right)$ but $\neg P(u, v, G)$, there is at least one birooted graph $G^{\star}{ }_{(u, v)} \in \mathcal{B}$ such that $G^{\star}{ }_{(u, v)} \subseteq G^{\prime}{ }_{(u, v)}$ but not $G^{\star}{ }_{(u, v)} \subseteq G_{(u, v)}$. Let $E=E\left(G^{\star}\right) \backslash E(G)$. That is, $E$ is a minimal set of edges that can be added to $G$ to establish $q$. (The set $E$ is minimal because $\mathcal{B}$ is assumed to be minimal.) The trace $\tau$ must contain a subsequence $\tau^{\prime}$ that correspond to a permutation of the edges in $E$. The subsequence $\tau^{\prime}$ is a minimal $(G, q)$-reduction of $\tau$. In fact, every $(G, q)$-reduction $\tau^{\prime}$ of $\tau$ corresponds to some permutation of an $E$ constructed as above from an appropriate choice of $G^{\star}$ (in the general case there may be multiple candidates for $\left.G^{\star}\right)$. Fixing one choice of $G^{\star}, E$ and $\tau^{\prime}$, we now show that there is a permutation $\pi$ of the edges in $E$ that is admissible. This permutation $\pi$ is a rationalization of $\tau^{\prime}$, as required by the theorem.

We construct $\pi$ as follows. As $N$ has SP, $P$ has SP at $u$. Let $v_{0}, v_{1}, \ldots, v_{n}=v$ and $G_{0}, G_{1}, \ldots, G_{n}=G^{\star}$ be the vertices and graphs promised by Definition 21. We start with $\pi$ being an empty trace. We will go through $n+1$ rounds of construction. In each round, we draw a number of (possibly zero) edges from $E$, and add them to the end of $\pi$. Specifically, no edge is selected in round 0 . Then, in round $i$, $i \in\{1, \ldots, n\}$, an edge $e$ is selected from $E$ iff one end of $e$ is $v_{i-1}$, and the other end of $e$ is one of $v_{i}, \ldots, v_{n}$. Multiple edges may be selected in the same round. In such a case, we add the edges to the end of $\pi$ in any arbitrary order. Note that every edge from $E$ is selected in exactly one round. Thus all edges in $E$ will have been added into $\pi$ by the end of the $n+1$ rounds.

We argue that $\pi$ is admissible. Let $\pi_{i}$ be the trace we have constructed by the end of round $i$. This trace $\pi_{i}$ is feasible in state $G$, as every transition in $\pi_{i}$ is a distinct befriending transition $e$ where $e$ is by construction absent from $E(G)$. Suppose $G \stackrel{\pi_{i}}{\longrightarrow}{ }_{N} G_{i}^{\sharp}$ for some social graph $G_{i}^{\sharp}$. We claim that $P\left(u, v_{i}, G_{i}^{\sharp}\right)$ is an invariant for the construction process. We prove this claim by first considering the case for $i=0$. Since $G_{0}^{\sharp}$ is obviously a supergraph of the empty graph, monotonicity and Lemma 26 guarantees that $P\left(u, v_{0}, G_{0}^{\sharp}\right)$ holds. We now consider the case when $i>0$. By monotonicity, the claim holds if $G_{i} \subseteq G_{i}^{\sharp}$. By SP, the vertex set $\left\{v_{0}, \ldots, v_{i-1}\right\}$ is a vertex cover of $E\left(G_{i}\right)$. This means every edge in $G_{i}$ either (a) is already in $G$, or (b) has already been added into $\pi_{i}$ in round $i$ or earlier. We thus have $G_{i} \subseteq G_{i}^{\sharp}$. So the claim holds. Now, we use the invariant to prove admissibility. Let $e$ be an arbitrary befriending transition in $\pi$, and let $i$ be the round in which $e$ is added into $\pi$. We know $i>0$ because no edge is selected in round 0 . By construction, one end of $e$ is $v_{i-1}$. We know from the invariant that $P\left(u, v_{i-1}, G_{i-1}^{\sharp}\right)$. When $\pi$ is executed from $G$, in the social graph $G^{b}$ immediately before $e$ is added, we shall have $P\left(u, v_{i-1}, G^{b}\right)$ by monotonicity (because $G_{i-1}^{\sharp} \subseteq$ $\left.G^{b}\right)$. Admissibility of $\pi$ is therefore guaranteed.

The second theorem to connect SP to POPA compliance is the completeness theorem.

Theorem 29 (Completeness). If an FSNS is POPA compliant, then it has SP.

Completeness asserts that the static analysis corresponding to SP is precise, in the sense that it does not miss out any opportunity to declare a system to be POPA compliant.

Proof: Suppose the FSNS $N=\langle S u b, P V, P o l\rangle$ is POPA compliant. We show that $N$ has SP. That is, we show that, for every user $u \in S u b$, the policy predicate $\operatorname{Pol}(u$, acc $)$ has SP at $u$.

Let $P$ be $\operatorname{Pol}(u$, acc $)$ for some arbitrary $u \in S u b$. Let $\mathcal{B}$ be the minimal set of birooted graphs to monotonically induce $P$. Consider an arbitrary member $G_{(u, v)}$ in $\mathcal{B}$. We construct for $G_{(u, v)}$ the sequences $v_{0}, v_{1}, \ldots, v_{n}=v$ and $G_{0}, G_{1}, \ldots$, $G_{n}=G$ as required by Definition 21 .

If $G$ is an empty graph, then the sequence can be constructed trivially by taking $n=0$.

Suppose $G$ contains at least one edge. Let $q$ be the query " $v$ reads $u$ ". Because $P$ is monotonic, and $\mathcal{B}$ is minimal, we know that no proper subgraph $G^{\prime}$ of $G_{0}$ can satisfy $P\left(u, v, G^{\prime}\right)$. Therefore, we have $G \vdash_{N} q$ but not $G^{\emptyset} \vdash_{N} q$. Let $\tau$ be a trace such that $G^{\emptyset} \stackrel{\tau}{\longrightarrow}{ }_{N} G$. The definition of POPA compliance (Definition 17) guarantees that every minimal $(G, q)$-reduction of $\tau$ has a rationalization. It is always possible to select $\tau$ so that $\tau$ is that rationalization.

Let $\tau=e_{0} e_{1} \ldots e_{m-1}$, where $m \geq 1$. Definition 15, together with the choice of $v$ and $G$, guarantee the existence of the vertex sequence $u_{0}, u_{1}, \ldots, u_{m-1}, u_{m}=v$, and the graph sequence $G^{\emptyset}=G_{0}^{\sharp}, G_{1}^{\sharp}, \ldots, G_{m-1}^{\sharp}, G_{m}^{\sharp}=G$ such that $G_{i}^{\sharp} \vdash_{N} u_{i}$ reads $u$ for $0 \leq i \leq m$. Note that the above vertex sequence may contain repeated occurrences of the same vertex, but the vertex $v$ occurs exactly once as the last vertex of the sequence (because $\tau$ is minimally reduced).

We construct in the following the sequence of distinct vertices $v_{0}, v_{1}, \ldots, v_{n}$ required by Definition 21 . Suppose there are $n+1$ distinct vertices in the sequence $u_{0}, u_{1}, \ldots$, $u_{m-1}, u_{m}$ (we know that $n \geq 1$, because $u_{m}$ is distinct from each of $u_{0}, \ldots, u_{m-1}$ and thus the sequence $u_{0}, u_{1}, \ldots$, $u_{m-1}, u_{m}$ contains at least two distinct vertices). We set $v_{i}$ to be $u_{j}$ such that $u_{j}$ is the $i$ 'th vertex to occur for the first time in the sequence. For example, if the sequence $u_{0}, u_{1}, \ldots$, $u_{m}$ is $a, a, c, a, b, c, a, d$, then $v_{0}=a, v_{1}=c, v_{2}=b$, and $v_{3}=d$. This choice is also consistent with the requirement 
that $v_{n}=v$. Let $G_{i}^{b}$ be the graph $G_{j}^{\sharp}$ such that $j$ is the smallest index for which $v_{i}=u_{j}$. Observe that the vertex set $V_{i}=\left\{v_{0}, \ldots, v_{i-1}\right\}$ is a vertex cover for $E\left(G_{i}^{b}\right)$. This is because Definition 15 guarantees that every edge in $G_{i}^{b}$ is added by a befriending transition initiated by some vertex in $V_{i}$. Since $P$ is monotonically induced by $\mathcal{B}$, there is a birooted graph $G_{i\left(u, v_{i}\right)} \in \mathcal{B}$ such that $G_{i} \subseteq G_{i}^{b} \subseteq G$. The vertex set $V_{i}$ must therefore be a vertex cover for $G_{i}$ as well.

Notice that the static analysis corresponding to Corollary 23, which examines the policy vocabulary rather than the actual access policies, is conservative (i.e., incomplete). A system with a policy vocabulary that contains SP-violating policies, can be POPA compliant so long as none of those SPviolating policies are actually adopted as access policies. In practice, however, Corollary 23 is the appropriate means for static analysis, as a system designer cannot anticipate which of the policies in the vocabulary are actually adopted.

The formulation of SP presented in this Section is very general, accommodating even policies that are not natural. The companion technical report [24] identifies a class of natural policies that we call proper policies. The report documents additional results regarding this class, including an alternative characterization of SP that is more convenient to use when the policies in question are proper.

Before we move on, the reader should be reminded that the results presented in this section apply only to an FSNS under Assumption 20. This means the results apply when we do not "blame" transitions in a trace that are responsible solely for enabling reachability of the owner's search listing, but not responsible for satisfying the owner's access policy.

\section{Mediated ACCESS}

In this section, we relax Assumption 20 (Unmediated Access), and devise a static analysis for the full model presented in Section III. That is, we use (R-ACC) rather than (R$\mathrm{ACC}^{-}$) to model access, and we no longer assume that $P V($ sch $)=\{\top\}$. Consequently, Stage-I authorization comes into full effect. In order to access the profile of user $u$, the accessor $v$ must first reach the search listing of $u$. That is, the query " $v$ finds $u$ " must be satisfied in order for access to be granted.

Incorporating Stage-I authorization into the analysis amounts to widening the scope of "blaming". Befriending transitions that establish the reachability of the owner's search listing, but otherwise play no role in satisfying the owner's access policy, are also "blamed" for enabling access. An important contribution of this section is to point out the analytical complexities and anomalies that could arise from this widening of "blaming" scope.

Let us formulate a high-level strategy of adapting the analysis of the previous section to this more general situation. A key observation is that, once the system $N$ is fixed, the sequent " $G \vdash_{N} \quad v$ finds $u$ ", as defined by the inference rules $(\mathrm{F}-\mathrm{SCH}),(\mathrm{F}-\mathrm{SLF}),(\mathrm{F}-\mathrm{FRD})$ and $(\mathrm{F}-\mathrm{TRV})$, induces a policy predicate that takes an owner $u$, an accessor $v$ and a social graph $G$, and returns a boolean value indicating reachability. Denote this predicate by $P_{\text {finds. }}$. (Note that $P_{\text {finds }}$ is monotonic but unlikely to be topology based, as each user may adopt a different search and traversal policy. This is one of the reasons our previous analyses are formulated in terms of monotonic rather than positive policies.) By (RACC), the sequent " $G \vdash_{N} v$ reads $u$ " holds whenever the policy predicate $P_{\text {finds }} \wedge \operatorname{Pol}(u$, acc $)$ is satisfied by $u, v$ and $G$. This is equivalent to the set-up in which Unmediated Access is assumed (i.e., $\left(\mathrm{R}-\mathrm{ACC}^{-}\right)$), but the access policy of $u$ is $P_{\text {finds }} \wedge \operatorname{Pol}(u$, acc $)$ instead. In other words, if one can show, through an appropriate static analysis, that $P_{\text {finds }} \wedge \operatorname{Pol}(u$, acc $)$ has SP at $u$, for each $u \in S u b$, then Theorem 28 guarantees that the system $N$ is POPA compliant. In addition, Theorem 29 guarantees that this analysis is precise. This is the approach to be taken in this section.

Corollary 30. Suppose $N=\langle S u b, P V$, Pol $\rangle$ is an FSNS. Then $N$ is POPA compliant iff the policy predicate $\left(P_{\text {finds }} \wedge\right.$ $\left.P_{\text {acc }}\right)$ has $S P$, where the predicate $P_{\text {finds }}(u, v, G)$ holds whenever $G \vdash_{N} v$ finds $u$, and the predicate $P_{\text {acc }}$ is defined as follows:

$$
P_{a c c}=\bigvee_{u \in S u b} \operatorname{Pol}(u, \text { acc })(u)
$$

There are two subproblems involved in assessing whether $\left(P_{\text {finds }} \wedge P_{\text {acc }}\right)$ has $\mathrm{SP}$. The first is to express $P_{\text {finds }}$ in terms of the search and traversal policies of $N$. In the companion technical report [24], we formulated a recurrence relation to facilitate the systematic derivation of $P_{\text {finds. }}$ In this paper, due to space limitation, we will derive $P_{\text {finds }}$ by first principle. The second subproblem is to show that the conjunction $\left(P_{\text {finds }} \wedge P_{\text {acc }}\right)$ has SP at every $u \in S u b$. Part of the problem has to do with identifying the condition under which conjunction preserves SP. It turns out that neither subproblem can always be solved, meaning that once we begin to "blame" transitions that are executed solely for the sake of establishing reachability as causal enablers of access, POPA compliance can no longer be guaranteed in many cases. In Sections VI-A and VI-B, we examine two instantiations of our model to illustrate how the above analytical approach unfolds. We then reflect on the proper interpretation of negative results in Section VI-C.

\section{A. Traversal Forbidden}

The first instantiation of the model that we consider corresponds to the following assumption.

Assumption 31. $P V($ tra $)=\{\perp\}$.

Under this assumption, the only traversal policy that can be adopted by any user is $\perp$. That is, the inference rule (F-TRV) is essentially rendered inapplicable. An accessor may reach the search listing of herself, a friend of hers, or someone reachable via global name search. Therefore, we have the following:

$$
P_{\text {finds }}=P_{\text {sch }} \vee \text { friend }
$$

where

$$
P_{\text {sch }}=\bigvee_{u \in S u b} \operatorname{Pol}(u, \text { sch })(u)
$$


That is, the FSNS is POPA compliant if we can demonstrate that, for every $u \in S u b$, the policy predicate $\left(P_{\text {sch }} \vee\right.$ friend $) \wedge$ $\operatorname{Pol}(u$, acc $)$ has SP at $u$. By Proposition 27.2, this holds when both $\left(P_{\text {sch }} \wedge \operatorname{Pol}(u\right.$, acc $\left.)\right)$ and $($ friend $\wedge \operatorname{Pol}(u$, acc $))$ have SP at $u$. We know friend has SP. Suppose we know also that all search and access policies have SP, can we then conclude that the two conjunctive policies above have SP? In general, conjunction does not preserve SP. A sufficient condition for SP to be preserved by conjunction is the following.

Definition 32. Let $\mathcal{B}_{1}, \mathcal{B}_{2}$ and $\mathcal{B}$ be the minimal sets of birooted graphs to monotonically induce $P_{1}, P_{2}$ and $P_{1} \wedge P_{2}$ respectively. Suppose further that $P_{1}$ and $P_{2}$ both have SP at $u$. Then $P_{1}$ and $P_{2}$, jointly, are said to have the Shared Substructure Property (SSP) at $u$ iff the following requirement holds:

Suppose $G_{(u, v)} \in \mathcal{B}_{i}$ is a birooted graph such that there is a corresponding birooted graph $G_{(u, v)}^{\prime} \in \mathcal{B}$ for which $G_{(u, v)} \subseteq G^{\prime}{ }_{(u, v)}$. (Such a $G_{(u, v)}$ is said to be realized.) The definition of $S P$ (Definition 21) guarantees the existence of a certain vertex sequence $v_{0}, v_{1}, \ldots, v_{n}=v$ and graph sequence $G_{0}, G_{1}, \ldots, G_{n}=G$. It is required that $G_{j\left(u, v_{j}\right)} \in$ $\mathcal{B}$ for $0 \leq j<n$.

In addition, if $P_{1}$ and $P_{2}$ have $S P$, then we say that they (jointly) have SSP when $P_{1}$ and $P_{2}$ have SSP at $u$ for every $u \in S u b$.

SSP is a property of an unordered pair of policy predicates. A few subtleties of the definition shall be highlighted. First, a birooted graph $G_{(u, v)}$ belonging to either $\mathcal{B}_{1}$ or $\mathcal{B}_{2}$ may not be contained in a member of $\mathcal{B}$ at all. This means that a birooted graph satisfying $P_{1} \wedge P_{2}$ may not contain $G_{(u, v)}$ as a birooted subgraph. Those that do are the ones that are "realized", and those are the ones that further requirements are imposed. Second, say $G_{(u, v)}$ belongs to one of $\mathcal{B}_{1}$ or $\mathcal{B}_{2}$. Because $P_{1}$ and $P_{2}$ have SP at $u$, the definition of SP guarantees that there is a vertex sequence $v_{0}, v_{1}, \ldots, v_{n}=v$ and graph sequence $G_{0}, G_{1}, \ldots, G_{n}=G$ that satisfy requirements (a), (b) and (c) of Definition 21. We now demand that the birooted graph $G_{j\left(u, v_{j}\right)}$ is not only a member of $\mathcal{B}_{i}$ (as required by Definition $21(\mathrm{~b}))$, but also a member of $\mathcal{B}$, the minimal set of birooted graphs to monotonically induce $P_{1} \wedge P_{2}$. This is demanded only for $0 \leq j<n$, meaning that we do not demand $G_{(u, v)}$ to be a member of $\mathcal{B}$.

Theorem 33. If policies $P_{1}$ and $P_{2}$ each has $S P$ (at $u$ ) and jointly have SSP (at $u$ ), then $P_{1} \wedge P_{2}$ has $S P$ (at $u$ ).

Note that the theorem asserts two statements. One with the "at $u$ " clauses present, and the other without. The theorem states that SP is preserved by conjunction when the two policies involved have SSP.

Proof: We prove the version of the theorem with the "at $u$ " clauses. The version without the "at $u$ " clauses follow immediately.

Suppose $P_{1}$ and $P_{2}$ each has SP at $u$ and jointly have SSP at $u$. Let $\mathcal{B}_{1}, \mathcal{B}_{2}$ and $\mathcal{B}$ be the minimal sets of birooted graphs to monotonically induce $P_{1}, P_{2}$ and $P_{1} \wedge P_{2}$ respectively. Our goal is to show that $P_{1} \wedge P_{2}$ has SP at $u$.

Consider $G_{(u, v)} \in \mathcal{B}$. By Proposition 10, $G_{(u, v)}$ can be expressed as $\left(G^{1} \cup G^{2}\right)_{(u, v)}$, where $G^{1}{ }_{(u, v)} \in \mathcal{B}_{1}$ and $G^{2}{ }_{(u, v)} \in \mathcal{B}_{2}$. Note that both $G^{1}{ }_{(u, v)}$ and $G^{2}{ }_{(u, v)}$ are realized. Definition 21 guarantees that there exists, for each $i \in\{1,2\}$, a vertex sequence $v_{0}^{i}, v_{1}^{i}, \ldots, v_{n_{i}}^{i}=v$ and a graph sequence $G_{0}^{i}, G_{1}^{i}, \ldots, G_{n_{i}}^{i}=G^{i}$, such that, for $0 \leq j \leq n_{i}$, we have: (a) $G_{j}^{i} \subseteq G^{i}$, (b) $G_{j\left(u, v_{j}^{i}\right)}^{i} \in \mathcal{B}_{i}$, and (c) the vertex set $V_{j}^{i}$ is a vertex cover for $E\left(G_{j}^{i}\right)$, where $V_{0}^{i}=\emptyset$, and $V_{j}^{i}=\left\{v_{0}^{i}, v_{1}^{i}, \ldots, v_{j-1}^{i}\right\}$ for $0<j \leq n$.

We now construct the required vertex sequence and graph sequence for $G_{(u, v)}$. Let $U=\left\{v_{0}^{1}, v_{1}^{1}, \ldots, v_{n_{1}}^{1}\right\} \cup$ $\left\{v_{0}^{2}, v_{1}^{2}, \ldots, v_{n_{2}}^{2}\right\}$. (Recall that $v_{n_{1}}^{1}=v_{n_{2}}^{2}=v$.) Let $n=$ $|U|$. We construct a vertex sequence $v_{0}, v_{1}, \ldots, v_{n}$ as follows. First, $v_{0}, v_{1}, \ldots, v_{n_{1}-1}$ are simply $v_{0}^{1}, v_{1}^{1}, \ldots, v_{n_{1}-1}^{1}$. Next, $v_{n_{1}}, v_{n_{1}+1}, \ldots, v_{n-1}$ are the vertices taken from $U \backslash\left\{v_{0}^{1}, v_{1}^{1}, \ldots, v_{n_{1}}^{1}\right\}$, in the order they appear in the vertex sequence $v_{0}^{2}, v_{1}^{2}, \ldots, v_{n_{2}}^{2}$. Lastly, $v_{n}=v$. We define the graph sequence $G_{0}, G_{1}, \ldots, G_{n}$ accordingly. First, the graphs $G_{0}, G_{1}, \ldots, G_{n_{1}-1}$ are $G_{0}^{1}, G_{1}^{1}, \ldots, G_{n_{1}-1}^{1}$. Next, the graphs $G_{n_{1}}, G_{n_{1}+1}, \ldots, G_{n-1}$ are those graphs among $G_{0}^{2}, G_{1}^{2}, \ldots, G_{n_{2}-1}^{2}$ that correspond to the vertices $v_{n_{1}}, v_{n_{1}+1}, \ldots, v_{n-1}$ selected out of $v_{0}^{2}, v_{1}^{2}, \ldots, v_{n_{2}-1}^{2}$. Lastly, we set $G_{n}$ to $G$. The following can be observed. First, $G_{j} \subseteq G$. This is because $G_{n}=G$ by construction, and $G_{j} \subseteq G^{i} \subseteq G^{1} \cup G^{2}=G$ for $0 \leq j<n$. Second, $G_{j\left(u, v_{j}\right)} \in \mathcal{B}$. In the case of $j=n, G_{n}=G \in \mathcal{B}$ by construction. In the case of $0 \leq j<n$, since $G_{n}^{i}$ is a realized member of $\mathcal{B}_{i}$, thus by SSP $G_{j} \in \mathcal{B}$. Third, the vertex set $V_{j}$ is a vertex cover for $E\left(G_{j}\right)$, where $V_{0}=\emptyset$, and, for $0<j \leq n$, $V_{j}=\left\{v_{0}, v_{1}, \ldots, v_{j-1}\right\}$. This follows immediately from the fact that $V_{j}^{i}$ is a vertex cover of $E\left(G_{j}^{i}\right)$.

Example 34. Suppose $P V($ acc $)=P V($ sch $)=\left\{\right.$ dist $_{k} \mid k \in$ $\mathbb{N}\}$. (Recall friend is but a shorthand for dist ${ }_{1}$.) Then every pair of such policies have both SP and SSP. The accessibility predicate $\left(P_{\text {sch }} \vee\right.$ friend $) \wedge \operatorname{Pol}(u$, acc $)$ has $S P$ at $u$, for every $u \in S u b$. By Corollary 30, the FSNS is POPA compliant.

While the instantiation above motivates SSP, the next instantiation illustrates the fact that we do not even need complete information of $P_{\text {finds }}$ in order to determine if the system is POPA compliant.

\section{B. Boolean Traversal Policies}

Let us apply our analysis to a slightly more complex FSNS, through a relaxation of Assumption 31.

Assumption 35. $P V(\operatorname{tra})=\{\perp, \top\}$.

Under this assumption, a user either allows every user to examine her friend list, or deny friend list access categorically. Although Assumption 35 appears to be a minor relaxation of Assumption 31, we shall see that it results in a major increase in the complexity of the analysis.

Our first order of business is to obtain a closed form for $P_{\text {finds. }}$. 
Suppose the FSNS is in state $G$. If there is a path $v_{0} v_{1} \ldots v_{n}$ in $G(n \geq 0)$ for which $\operatorname{Pol}\left(v_{i}\right.$, tra $)=\top$ holds for $0<i \leq n$, then we say that the path is a $\top$-path from $v_{0}$ to $v_{n}$. Note that we do not require that $\operatorname{Pol}\left(v_{0}\right.$, tra $)=\top$. Also, a length-zero path (i.e., $n=0$ ) is always a $\top$-path. The intuition behind the definition of a $T$-path is this: if a user can reach the search listing of $v$, then that user can further reach the search listing of $u$ through $n$ applications of (F-TRV) whenever there is a $\top$-path from $u$ to $v$. At the heart of the above observation is that, under Assumption 35, what determines whether $v$ can traverse through the friend list of $u$ is not the whereabout of $v$ in relation to $u$, but the setting of $\operatorname{Pol}(u$, tra $)$ as $\perp$ or $\top$.

Define the policy predicate traverse such that traverse $(u, v, G)$ holds whenever there is a T-path (of any length) from $u$ to $v$ in $G$. Note that traverse is a monotonic predicate. More importantly, since every subpath of a $\top$-path is also a $\top$-path, it is easy to show that traverse has SP.

The following closed form for $P_{\text {finds }}$ can be shown:

$$
P_{\text {finds }}=\text { traverse } \circ\left(P_{\text {sch }} \vee \text { friend }\right)
$$

Intuitively, a search listing can be reached by applying one of (F-SCH), (F-SLF) or (F-FRD), followed by applying (F-TRV) for zero or more times.

Suppose we know that $P V$ (sch) contains only policies that have SP, and thus $P_{\text {sch }}$ has SP. We still cannot conclude that $P_{\text {finds }}=$ traverse $\circ\left(P_{\text {sch }} \vee\right.$ friend $)$ has SP because SP is not preserved by the $\circ$ combinator. The following example shows us that in some cases, we may still show that $P_{\text {finds }}$ has SP, but this is not true in general.

Example 36. Suppose $P V($ sch $)=\{\perp$, connected,$\top\}$, where the predicate connected holds whenever there is a path between the owner and the accessor. (Note that connected has SP.) Consider $P_{\text {finds }}(u)$. There are three cases. First, if there is a T-path from $u$ to a vertex $u^{\prime}$ for which $\operatorname{Pol}\left(u^{\prime}, s c h\right)=\top$, then every user $v$ can reach the search listing of $u$ by first reaching $u^{\prime}$ by global name search (which will always succeed), and then traversing the $T$-path to reach $u$. Therefore, $P_{\text {finds }}(u)=\top(u)$. Second, if the first case does not apply, but there is a T-path from $u$ to a vertex $u^{\prime}$ for which $\operatorname{Pol}\left(u^{\prime}\right.$, sch $)=$ connected, then every user $v$ which is connected to $u^{\prime}$ can reach the search listing of $u$. But these $v$ are exactly those that are connected to $u$ itself. Therefore, $P_{\text {finds }}(u)=$ connected $(u)$. Third, if neither the first nor the second case applies, then every vertex $u^{\prime}$ reachable from $u$ via a T-path is such that $\operatorname{Pol}\left(u^{\prime}, s c h\right)=\perp$. In this case, either $u^{\prime}$ or a friend of $u^{\prime}$ can reach the search listing of $u$. Thus, $P_{\text {finds }}(u)=$ traverse $\circ$ friend. In summary, $P_{\text {finds }}(u) \in\{($ traverse $\circ$ friend $)(u)$, connected $(u), \top(u)\}$.

Let us consider two instantiations of this FSNS.

1) $P V($ acc $)=\{\perp$, connected, $\top\}$. Then it can be shown that $P_{\text {finds }}(u) \wedge \operatorname{Pol}(u$, acc $) \in\{\perp$, (traverse $\circ$ friend $(u)$, connected $(u), \top(u)\}$ has SP. Thus the FSNS is POPA compliant.
2) $P V($ acc $)=\left\{\right.$ dist $\left._{k} \mid k \in \mathbb{N}\right\}$. Then it is possible that $P_{\text {finds }}(u) \wedge \operatorname{Pol}(u$, acc $)=$ traverse $(u) \wedge$ dist $_{k}$ for some $k \in \mathbb{N}$, which can be shown not to have SP. (It is easy to show that traverse and dist $t_{k}$ do not have SSP.) Thus the FSNS is not POPA compliant.

Observe that our goal above is not to obtain a precise closed form for $\left(P_{\text {finds }} \wedge P_{\text {acc }}\right)$ : we do not even know what traverse looks like. Instead, the main goal is to identify the major subexpressions in $\left(P_{\text {finds }} \wedge P_{\text {acc }}\right)$, so that we know to which policies and policy pairs we shall impose SP and SSP respectively.

\section{Interpretation of Negative Results}

The second instantiation of Example 36 illustrates that there are in fact FSNSs for which all search, traversal and access policies have SP, but the FSNSs themselves are not POPA compliant. The reason for this is that we now demand the conjunction $\left(P_{\text {finds }} \wedge P_{\text {acc }}\right)$ to have SP (Corollary 30), rather than requiring simply that $P_{\text {acc }}$ has SP (Theorem 28). The failure of the conjunction to satisfy the requirements of SP may be caused by at least two reasons. First, if the reachability predicate $P_{\text {finds }}$ has SP, the requirement of SSP may not be satisfied by the relevant policy pairs (e.g., second instantiation of Example 36). Second, $P_{\text {finds }}$ may not have SP. The structure of Stage-I authorization is designed in such a way that SP is not a condition we can expect to hold by $P_{\text {finds }}$ except for a few special cases. In fact, as the next example illustrates, so long as $P V$ (tra) contains non-constant predicates, it is unlikely that $P_{\text {finds }}$ has SP.

Example 37. Suppose $P V($ sch $)=P V($ tra $)=P V($ acc $)=$ $\{\perp$, me, friend, fof $\top\}$. (Note the resemblance between this policy vocabulary and that of Facebook.) $P_{\text {acc }}$ has $S P$ (Example 25), but neither $P_{\text {finds }}$ nor $P_{\text {finds }} \wedge P_{\text {acc }}$ has $S P$.

This situation demands us to be very careful in interpreting negative results. Suppose $N$ is an FSNS for which $P_{\text {acc }}$ has SP, but $\left(P_{\text {finds }} \wedge P_{\text {acc }}\right)$ does not have SP. By Corollary 30, we would conclude that $N$ is not POPA compliant, and thus Sybil attacks are possible in $N$ (Theorem 19). But then exactly what is the nature of these Sybil attacks? It turns out such a Sybil attack is caused by befriending transitions which (i) establish the reachability of the owner's search listing, (ii) play no role in satisfying the owner's access policy, and (iii) involve no initiator that already has access. Yet the assumption is that the access policies have SP. This means in the start state of the Sybil attack, the accessor and her colluding partners already satisfy the access policy of the owner. The reason they do not have access to begin with is because they cannot reach the owner's search listing in the start state. That means all that the offending transitions achieve is to turn the owner's search listing from unreachable to reachable by the accessor and her colluding partners.

The crux of the problem is how we are to perceive Stage-I Authorization. Suppose we do not consider Stage-I Authorization to be a proper component of access authorization 
(Section V-A). That is, friend lists are no different than other profile items, and thus traversal policies are merely access policies for friend lists. Then so long as we demand that the traversal policies have SP, the FSNS is POPA compliant, and the above anomaly disappears. Suppose, however, we consider Stage-I Authorization to be a proper component of access authorization, such that the search listings are capability-like entities, the reachability of which is a necessary condition for access. The analysis of this section implies that, except in a few special cases, POPA compliance is not achievable. Our conclusion is that the current design of Stage-I Authorization does not support a rational static analysis for POPA compliance. As we will point out in Section VIII, a redesign of StageI Authorization to facilitate POPA-compliance analysis is a pressing research challenge.

\section{RELATED WORK}

Recent years have seen the proposal of novel authorization schemes for Relationship-Based Access Control (ReBAC) [3], [4], [5], [6]. In the access control models of Kurk et al. [25] and Carminati et al. [26], [27], access control policies identify the existence of a directed path between the owner and the accessor in the social network, consisting of edges of a particular relationship type, within a certain length, and with aggregate trust weighting above a certain threshold. Carminati et al. [28], [29], [30] later refined their model to use a trust metric for decentralized authorization. In [31], [32], Carminati et al employed Semantic Web technologies to encode access control information of a ReBAC system. Fong et al. [7], [8] proposed a formal model for Facebook-style Social Network Systems (FSNSs), which is a generalization of the access control model behind Facebook. Although this model does not support directed relationships, relationship types, and trust levels, it supports a uniquely novel feature missing from previously proposed models, namely, the use of arbitrary graph-theoretic relations as access control policies. Fong [5] later devised a ReBAC model that adds support for relationship types and access contexts on top of graph-theoretic policies. A modal logic is employed as its policy language, rather than birooted graphs. The expressiveness of this policy language and its extension are studied in [6]. Squicciarini et al. [33] proposed a scheme for automatically deriving access control policies for the users of an SNS. The present work is an attempt to identify a security property, namely, Principle of Privilege Attenuation, that the above authorization schemes shall strive to guarantee.

To the best of our knowledge, this work is the first to propose a policy analysis for verifying that an SNS complies to a global security property. Comparable to our goal is the work of Squicciarini et al. [34], [35], which studied the collaborative management (or co-ownership) of resources in SNSs. Gametheoretic analysis is employed to ensure fairness and other global properties in their protocol.

Traditional defences against Sybil attacks begin with (direct) identity validation [9], [36], [37], [38], the goal of which is to prevent large-scale injection of pseudonymous identities by a small number of malicious users. Douceur [9] proposed the use of computational challenges to validate that the user behind an identity indeed possesses certain computational resources (e.g., communication, storage, computing power). A malicious user forging multiple, pseudonymous identities will fail to meet these challenges. Similar use of client puzzles for admission control were found in [36], [37], [38]. CAPTCHA [39] is a popular variation of this approach to tell human users apart from computational agents. In the light of the feasibility of Profile Cloning attacks [10], [11], [12] in popular SNSs (including Facebook), partly made possible by circumventing CAPTCHA, this work accepts as an axiom that pseudonymous identities are not avoidable in FSNS. Instead, the goal of this work is to explore how an FSNS can be designed to prevent Sybil attacks even in the presence of pseudonymous identities.

In recent years, major headway has been made to develop near-optimal or optimal defence mechanisms against Sybil attacks in peer-to-peer networks [13], [14] and recommendation systems [16]. Particularly notable are SybilGuard [13] and its successor SybilLimit [14], which provide randomized protocols for a node (the verifier) in a peer-to-peer system to determine if it should engage in a transaction with another node (the suspect). The verification step of both protocols involves performing randomized routing in a stationary social graph defined over all nodes. Exploiting a certain property ("fast-mixing") of social graphs, one can guarantee that, with arbitrarily high probability, the verifiers will accept only logarithmically many Sybil nodes, and turn away arbitrary few honest nodes. A recommendation system rates objects based on the previous assessment of these objects by a population of users. Sybil attacks occur when a number of malicious users or pseudonymous identities collude to influence object ratings. DSybil [16] is a recommendation system that comes with recommendation quality guarantee even in the presence of Sybil attacks. Specifically, an upper bound for bad recommendations made by the system in the worst case is established alongside a matching lower bound. The above works are related to our work in that they all accept pseudonymous identities as an unavoidable reality, and provide protection against Sybil attacks, with provable guarantee in the quality of protection. The similarity, however, ends here. First, our work studies Sybil attacks in the novel context of a RelationshipBased Access Control system (i.e., FSNS), rather than peerto-peer or recommendation systems. Our goal is to prevent pseudonymous identities from manipulating the topology of the social graph in order to gain access. Second, our solution approach is a static analysis on the policy vocabulary, from which users may freely adopt their own policies, rather than the imposition of a single policy. Third, our guarantee (Sybil freedom via a soundness theorem) is absolute rather than probabilistic, and is not based on assumptions regarding the topology of social graphs.

Cheng and Friedman [15] studied characteristics of reputation functions that are Sybilproof. A reputation function maps an edge-weighted graph and a vertex to a reputation value for that vertex. They showed that Sybilproof reputation functions 
cannot be "symmetric", meaning that the reputation value is invariant under graph isomorphism. Their result does not apply to our topology-based policies, for which the authorization decision is invariant under birooted-graph isomorphism ${ }^{4}$.

\section{SUMMARY AND FUTURE WORK}

In this work, we examined the challenge of preventing Sybil attacks in Facebook-style Social Network Systems. We formulated a version of the Principle of Privilege Attenuation for FSNSs, and showed that it is the necessary and sufficient condition for preventing Sybil attacks. We then devised a static policy analysis for verifying if an FSNS is POPA compliant. The analysis is both sound and complete. Lastly, we demonstrated the complexities and anomalies that would arise from incorporating Stage-I Authorization into the analysis.

To address the anomalies surrounding Stage-I Authorization, two research questions present themselves. The first is to devise a better characterization of those policy vocabularies that guarantee POPA compliance even in the presence of Stage-I Authorization. The second is to redesign Stage-I Authorization to facilitate a rational POPA-compliance analysis.

The results in this work apply only to monotonic policies. A future direction is to explore how the analysis can be extended to cover policies that may not be monotonic. Also implicit in the current work is the assumption of a particular consent protocol: befriending requires the consent of both parties, but defriending can be initiated unilaterally. Our preliminary experience with nonmonotonic policies suggests that alternative consent protocols may be required in order for the static analysis to become feasible in the midst of nonmonotonic policies.

While this work focuses on static policy analysis, a natural question is whether POPA compliance can be achieved by coupling static analysis of policy vocabulary with dynamic checks performed at run time. A challenge will be to minimize the run-time and storage overhead required for such a scheme.

In this work, Sybil attack prevention is taken as a security goal of ReBAC. It is worthwhile to explore alternative security goals for ReBAC, or relaxations of the present security goal. An example of the latter option is to impose lower bound for the "cost" of launching a Sybil attack (e.g., the number of colluding users required [40], etc).

\section{ACKNOWLEDGMENT}

This work is funded in part by an NSERC Strategic Project Grant, and has benefited from the in-kind support of Security Resource Group Inc.

\section{REFERENCES}

[1] J. A. Goguen and J. Meseguer, "Security policies and security models," in Proceedings of the 1982 IEEE Symposium on Security and Privacy (S\&P'82), 1982, pp. 11-20.

[2] J. Rushby, "Noninterference, transitivity, and channel-control security policies," Computer Science Laboratory, SRI International, Tech. Rep. CSL 92-02, 1992.

${ }^{4}$ It is also worthwhile to point out that there is a flaw in the proof of their Theorem 1, which incorrectly claims that the social graphs before and after a Sybil attack are isomorphic.
[3] C. E. Gates, "Access control requirements for Web 2.0 security and privacy," in IEEE Web 2.0 privacy and security workship (W2SP'07), Oakland, California, USA, May 2007.

[4] B. Carminati and E. Ferrari, "Enforcing relationships privacy through collaborative access control in web-based social networks," in Proceedings of the 5th International Conference on Collaborative Computing: Networking, Applications and Worksharing (CollaborateCom'09), Washington DC, USA, Nov. 2009.

[5] P. W. L. Fong, "Relationship-based access control: protection model and policy language," in Proceedings of the First ACM Conference on Data and Application Security and Privacy (CODASPY'11), San Antonio, TX, USA, Feb. 2011, pp. 191-202.

[6] P. W. L. Fong and I. Siahaan, "Relationship-based access control policies and their policy languages," in Proceedings of the 16th ACM Symposium on Access Control Models and Technologies (SACMAT'11), Innsbruck, Austria, Jun. 2011.

[7] P. W. L. Fong, M. Anwar, and Z. Zhao, "A privacy preservation model for Facebook-style social network systems," in Proceedings of the 14th European Symposium on Research In Computer Security (ESORICS'09), ser. LNCS, vol. 5789. Saint Malo, France: Springer, Sep., pp. 303-320.

[8] M. Anwar, Z. Zhao, and P. W. L. Fong, "An access control model for Facebook-style social network systems," Department of Computer Science, University of Calgary, Calgary, Alberta, Canada, Tech. Rep. 2010-959-08, Jul. 2010, submitted for review.

[9] J. R. Douceur, "The Sybil attack," in Proceedings for the First International Workshop on Peer-to-Peer Systems (IPTPS'02), ser. LNCS, vol. 2429. Cambridge, MA, USA: Springer, Mar. 2002, pp. 251-260.

[10] L. Bilge, T. Strufe, D. Balzarotti, and E. Kirda, "All your contacts are belong to us: Automated identity theft attacks on social networks," in Proceedings of the 18th International Conference on World Wide Web (WWW'09), Madrid, Spain, Apr. 2009, pp. 551-560.

[11] L. Jin, H. Takabi, and J. B. D. Joshi, "Towards active detection of identity clone attacks on online social networks," in Proceedings of the First ACM Conference on Data and Application Security and Privacy (CODASPY'11), San Antonio, TX, USA, Feb. 2011, pp. 27-38.

[12] G. Kontaxis, I. Polakis, S. Ioannidis, and E. P. Markatos, "Detecting social network profile cloning," in Proceedings of the 3rd IEEE International Workshop on Security and Social Networking (SESOC'11), Seattle, WA, USA, Mar. 2011.

[13] H. Yu, M. Kaminsky, P. B. Gibbons, and A. D. Flaxman, "SybilGuard: Defending against Sybil attacks via social networks," IEEE/ACM Transactions on Networking, vol. 16, no. 3, pp. 576-589, Jun. 2008.

[14] H. Yu, P. B. Gibbons, M. Kaminsky, and F. Xiao, "SybilLimit: A nearoptimal social network defense against Sybil attacks," in Proceedings of the 2008 IEEE Symposium on Security and Privacy (S\&P'08), Oakland, CA, USA, May 2008, pp. 3-17.

[15] A. Cheng and E. Friedman, "Sybilproof reputation mechanisms," in Proceedings of the 2005 ACM SIGCOMM Workshop on Economics of peer-to-peer systems (P2PEcon'05), Philadelphia, PA, USA, Aug. 2005, pp. $128-132$.

[16] H. Yu, C. Shi, M. Kaminsky, P. B. Gibbons, and F. Xiao, "DSybil: Optimal Sybil-resistance for recommendation systems," in Proceedings of the 2009 IEEE Symposium on Security and Privacy (S\&P'09), Berkeley, CA, USA, May 2009, pp. 283-298.

[17] J. B. Dennis and E. C. V. Horn, "Programming semantics for multiprogrammed computations," Communications of the ACM, vol. 9, no. 3, pp. 143-155, Mar. 1966.

[18] M. S. Miller, K.-P. Yee, and J. Shapiro, "Capability myths demolished," System Research Lab, Department of Computer Science, The John Hopkins University, Baltimore, Maryland, USA, Tech. Rep. SRL200302, 2003.

[19] G. S. Graham and P. J. Denning, "Protection: Principles and practices," in Proceedings of the 1972 AFIPS Spring Joint Computer Conference, vol. 40, Alantic City, New Jersey, USA, May 1972, pp. 417-429.

[20] N. Li and M. V. Tripunitara, "On safety in discretionary access control," in Proceedings of the 2005 IEEE Symposium on Security and Privacy (S\&P'05), Oakland, California, USA, May 2005, pp. 96-109.

[21] P. J. Denning, "Fault tolerant operating systems," ACM Computing Surveys, vol. 8, no. 4, pp. 359-389, Dec. 1976.

[22] M. Bishop, Computer Security. Addison Wesley, 2002

[23] M. R. Clarkson and F. B. Schneider, "Hyperproperties," in Proceedings of the 21st IEEE Computer Security Foundations Symposium (CSF'08), Pittsburch, Pennsylvania, USA, Jun. 2008, pp. 51-65. 
[24] P. W. L. Fong, "Preventing Sybil attacks by privilege attenuation: A design principle for social network systems," Department of Computer Science, University of Calgary, Calgary, Alberta, Canada, Tech. Rep. 2011-995-07, Mar. 2011.

[25] S. R. Kruk, S. Grzonkowski, A. Gzella, T. Woroniecki, and H.-C. Choi, "D-FOAF: Distributed identity management with access rights delegation," in Proceedings of the First Asian Semantic Web Conference (ASWC'06), ser. LNCS, vol. 4185. Beijing, China: Springer, Sep. 2006, pp. 140-154.

[26] B. Carminati, E. Ferrari, and A. Perego, "Rule-based access control for social networks," in Proceedings of the OTM 2006 Workshops, ser. LNCS, vol. 4278. Springer, Oct. 2006, pp. 1734-1744.

[27] — "A decentralized security framework for web-based social networks," International Journal of Information Security and Privacy, vol. 2 , no. 4, pp. 22-53, Oct. 2008.

[28] — , "Private relationships in social networks," in Proceedings of Workshops in Conjunction with the International Conference on Data Engineering - ICDE'07, Istanbul, Turkey, Apr. 2007, pp. 163-171.

[29] B. Carminati and E. Ferrari, "Privacy-aware collaborative access control in web-based social networks," in Proceedings of the 22nd Annual IFIP WG 11.3 Working Conference on Data and Applications Security (DAS'08), ser. LNCS, vol. 5094. London, UK: Springer, Jul. 2008, pp. 81-96.

[30] B. Carminati, E. Ferrari, and A. Perego, "Enforcing access control in web-based social networks," ACM Transactions on Information and System Security, vol. 13, no. 1, Oct. 2009.

[31] B. Carminati, E. Ferrari, R. Heatherly, M. Kantarcioglu, and B. Thurainsingham, "A semantic web based framework for social network access control," in Proceedings of the 14th ACM Symposium on Access Control Models and Technologies (SACMAT'09), Stresa, Italy, Jun. 2009, pp. 177-186.

[32] B. Carminati, E. Ferrari, R. Heatherly, M. Kantarcioglu, and B. Thuraisingham, "Semantic web-based social network access control," Computers and Security, vol. 30, no. 2-3, pp. 108-115, Mar. 2011.

[33] A. Squicciarini, F. Paci, and S. Sundareswaran, "PriMa: An effective privacy protection mechanism for social networks," in Proceedings of the 5th ACM Symposium on Information, Computer and Communications Security (ASIACCS'10), Beijing, China, Apr. 2010, pp. 320-323.

[34] A. C. Squicciarini, M. Shehab, and F. Paci, "Collective privacy management in social networks," in Proceedings of the 18th International Conference on World Wide Web (WWW'09), Madrid, Spain, Apr. 2009, pp. 521-530.

[35] A. C. Squicciarini, M. Shehab, and J. Wede, "Privacy policies for shared content in social network sites," The VLDB Journal, 2010, to appear.

[36] M. Castro, P. Druschel, A. Ganesh, A. Rowstron, and D. S. Wallach, "Secure routing for structured peer-to-peer overlay networks," in Proceedings of the 5th USENIX Symposium on Operating Systems Design and Implementation (OSDI'02), Boston, MA, USA, Dec. 2002.

[37] N. Borisov, "Computational puzzles as sybil defenses," in Proceedings of the 6th IEEE International Conference on Peer-to-Peer Computing (P2P'06), Cambridge, UK, Sep. 2006, pp. 171-176.

[38] H. Rowaihy, W. Enck, P. McDaniel, and T. L. Porta, "Limiting Sybil attacks in structured P2P networks," in Proceedings of the 26th IEEE International Conference on Computer Communications (INFOCOM'07), Anchorage, Alaska, USA, May 2007, pp. 2596-2600.

[39] L. von Ahn, M. Blum, N. J. Hopper, and J. Langford, "CAPTCHA Using hard AI problems for security," in Proceedings of the 22nd International Conference on the Theory and Applications of Cryptographic Techniques (EUROCRYPT'03), ser. LNCS, vol. 2656. Warsaw, Poland: Springer, May 2003, pp. 294-311.

[40] L. Snyder, "Theft and conspiracy in the take-grant protection model," Journal of Computer and System Sciences, vol. 23, no. 3, pp. 333-347, 1981. 